\title{
Information Transmission using the Nonlinear Fourier Transform, Part II: Numerical Methods
}

\author{
Mansoor I. Yousefi and Frank R. Kschischang, Fellow, IEEE
}

\begin{abstract}
In this paper, numerical methods are suggested to compute the discrete and the continuous spectrum of a signal with respect to the Zakharov-Shabat system, a Lax operator underlying numerous integrable communication channels including the nonlinear Schrödinger channel, modeling pulse propagation in optical fibers. These methods are subsequently tested and their ability to estimate the spectrum are compared against each other. These methods are used to compute the spectrum of various signals commonly used in the optical fiber communications. It is found that the layer-peeling and the spectral methods are suitable schemes to estimate the nonlinear spectra with good accuracy. To illustrate the structure of the spectrum, the locus of the eigenvalues is determined under amplitude and phase modulation in a number of examples. It is observed that in some cases, as signal parameters vary, eigenvalues collide and change their course of motion. The real axis is typically the place from which new eigenvalues originate or are absorbed into after traveling a trajectory in the complex plane.
\end{abstract}

Index Terms-Optical fiber communication, forward nonlinear Fourier transform, Zakharov-Shabat spectral problem, numerical methods, operator eigenproblem.

\section{INTRODUCTION}

$\mathbf{T}$ HE nonlinear Fourier transform (NFT) of a signal $q(t)$ is a pair of functions: the continuous spectrum $\hat{q}(\lambda), \lambda \in \mathbb{R}$, and the discrete spectrum $\tilde{q}\left(\lambda_{j}\right), \Im \lambda_{j}>0, j=1, \ldots, \mathrm{N}$. The NFT arises in the study of integrable waveform channels as defined in Part I [1]. In such channels, signals propagate (in a potentially complicated manner) according to a given integrable evolution equation, whereas the nonlinear Fourier transform of the signal propagates according to a (simple) multiplication operator.

In [Part I], we proposed nonlinear frequency-division multiplexing (NFDM), a scheme that uses the nonlinear Fourier transform for data communication over integrable channels. NFDM extends traditional orthogonal frequency-division multiplexing (OFDM) to channels generatable by a Lax pair. An example is the optical fiber channel, where signal propagation is modeled by the (integrable) nonlinear Schrödinger (NLS) equation. In general, the channel input-output relations in the NFT domain are (see [Part I])

$$
\begin{aligned}
\hat{Y}(\lambda) & =H(\lambda) \hat{X}(\lambda)+\hat{Z}, \\
\tilde{Y}\left(\lambda_{j}\right) & =H\left(\lambda_{j}\right) \tilde{X}\left(\lambda_{j}\right)+\tilde{Z},
\end{aligned}
$$

Submitted for publication on April 3, 2012; accepted December 14, 2012. The authors are with the Edward S. Rogers Sr. Dept. of Electrical and Computer Engineering, University of Toronto, Toronto, ON M5S 3G4, Canada, e-mails: \{mansoor,frank\}@ comm.utoronto.ca. where $\hat{X}(\lambda)$ and $\tilde{X}\left(\lambda_{j}\right)$ are continuous and discrete spectra at the input of the channel, $\hat{Y}(\lambda)$ and $\tilde{Y}\left(\lambda_{j}\right)$ are spectra at the output of the channel, and $\hat{Z}$ and $\tilde{Z}$ represent noise. The channel filter $H(\lambda)$ for the NLS equation is given by $H(\lambda)=$ $\exp \left(-4 j \lambda^{2} z\right)$.

NFDM is able to deal directly with nonlinearity and dispersion, without the need for additional compensation at the transmitter or receiver. In this scheme, information is encoded in the nonlinear spectrum at the channel input, and the corresponding time-domain signal is transmitted. At the receiver, the NFT of the received signal is computed, and the resulting spectra $\hat{Y}(\lambda)$ and $\tilde{Y}\left(\lambda_{j}\right)$ are subsequently used to recover the transmitted information.

Similar to the ordinary Fourier transform, while the NFT can be computed analytically in a few cases, in general, numerical methods are required. Such methods must be robust, reliable and fast enough to be implemented in real-time at the receiver. In this paper, we suggest and evaluate the performance of a number of numerical algorithms for computing the forward NFT of a given signal. Using these algorithms, we then perform extensive numerical simulations to understand the behavior of the nonlinear spectrum for various pulse shapes and parameters commonly used in data communications.

We are aware of no published work presenting the NFT of various signals numerically, for many pulse shapes and parameters. Such work is necessary to clarify the structure of the nonlinear spectrum and help in its understanding. In part, this has been due to the fact that the NFT has largely remained a theoretical artifice, and practical implementation of the NFT as an applied tool has not yet been pursued in engineering.

We review the relevant literature in Section $\Pi$ and suggest new schemes for the numerical evaluation of the forward NFT. Although these methods are general and work for the AKNS system [2], for the purpose of illustration, we specialize the AKNS system to the Zakharov-Shabat system. All these methods are put to test in cases where analytical formulae exist and are compared with one another in Section VI. Only some of these methods will be chosen for the subsequent numerical simulations; these are the layer-peeling method, AblowitzLadik integrable discretization, and the spectral matrix eigenvalue scheme. These methods are used in the next sections to numerically compute the nonlinear Fourier transform of a variety of practical pulse shapes encountered in the data communications. 


\section{The NONLINEAR Fourier TRANSFORM}

Details of the nonlinear Fourier transform can be found in [Part I]. Here we briefly recall a few essential ingredients required in the numerical computation of the forward transform. As noted earlier, we illustrate numerical methods in the context of the Zakharov-Shabat system, which is a Lax operator for the nonlinear Schrödinger equation.

For later use, we recall that the slowly-varying complex envelope $q(t, z)$ of a narrow-band small-amplitude signal propagating in a dispersive weakly-nonlinear medium, such as an optical fiber, satisfies the cubic nonlinear Schrödinger equation. By proper scaling, the equation can be normalized to the following dimensionless form in $1+1$ dimensions:

$$
j q_{z}=q_{t t}+2|q|^{2} q .
$$

Here $t$ denotes retarded time, and $z$ is distance.

The NFT for an integrable evolution equation starts by finding a Lax pair of operators $L$ and $M$ such that the evolution equation arises as the compatibility condition $L_{z}=$ $[M, L]=M L-L M$. For the NLS equation, we may take operator $L$ as

$$
L=j\left(\begin{array}{cc}
\frac{\partial}{\partial t} & -q(t, z) \\
-q^{*}(t, z) & -\frac{\partial}{\partial t}
\end{array}\right) .
$$

(The corresponding $M$ operator can be found in [Part I].)

The NFT is defined via the spectral analysis of the $L$ operator, given in this paper by (2). The spectrum of $L$ is found by solving the eigenproblem $L v=\lambda v$, where $\lambda$ is an eigenvalue of $L$ and $v$ is its associated eigenvector. It can be shown that the operator $L$ in (2) has the isospectral flow property, i.e., its spectrum is invariant even as $q$ evolves according to the NLS equation.

The eigenproblem $L v=\lambda v$ can be simplified to

$$
v_{t}=\left(\begin{array}{cc}
-j \lambda & q(t) \\
-q^{*}(t) & j \lambda
\end{array}\right) v .
$$

Note that the $z$-dependence of $q$ is suppressed in (3) (and throughout this paper), as this variable comes into play only in the propagation of the signal, not in the definition and computation of the NFT.

Assumption 1. Throughout this paper we assume that (a) $q \in L^{1}(\mathbb{R})$, and $(b) q(t)$ is supported in the finite interval $\left[T_{1}, T_{2}\right]$.

The set of eigenvectors $v$ associated with eigenvalue $\lambda$ in (3) is a two-dimensional subspace $E_{\lambda}$ of the continuously differentiable functions. We define the adjoint of a vector $v=\left[v_{1}(t), v_{2}(t)\right]^{T}$ as $\tilde{v}=\left[v_{2}^{*}(t),-v_{1}^{*}(t)\right]^{T}$. If $v\left(t, \lambda^{*}\right)$ is an element of $E_{\lambda^{*}}$, then $\tilde{v}\left(t, \lambda^{*}\right)$ is an element of $E_{\lambda}$. It can be shown that any pair of eigenvectors $v(t, \lambda)$ and $\tilde{v}\left(t, \lambda^{*}\right)$ forms a basis for $E_{\lambda}$ [Part I]. Using Assumption 11b), we can select an eigenvector $v^{1}(t, \lambda)$ to be a solution of (3) with the boundary condition

$$
v^{1}\left(T_{2}, \lambda\right)=\left(\begin{array}{l}
0 \\
1
\end{array}\right) e^{j \lambda T_{2}} .
$$

The basis eigenvectors $v^{1}$ and $\tilde{v}^{1}$ are called canonical eigenvectors.
Having identified a basis for the subspace $E_{\lambda}$, we can project any other eigenvector $v^{2}$ on this basis according to

$$
v^{2}(t, \lambda)=a(\lambda) \tilde{v}^{1}(t, \lambda)+b(\lambda) v^{1}(t, \lambda) .
$$

Following Assumption 1(b), a particular choice for $v^{2}$ is made by solving the system

$$
v_{t}=\left(\begin{array}{cc}
-j \lambda & q(t) \\
-q^{*}(t) & j \lambda
\end{array}\right) v, \quad v\left(T_{1}, \lambda\right)=\left(\begin{array}{l}
1 \\
0
\end{array}\right) e^{-j \lambda T_{1}},
$$

in which we dropped the superscript 2 in $v^{2}$ for convenience. By solving (5) in the interval $\left[T_{1}, T_{2}\right]$ for a given $\lambda$ and obtaining $v\left(T_{2}, \lambda\right)$, the nonlinear Fourier coefficients $a(\lambda)$ and $b(\lambda)$ can be obtained by considering (4) at $t=T_{2}$. The resulting coefficients obtained in this manner are

$$
\begin{aligned}
a(\lambda) & =v_{1}\left(T_{2}\right) e^{j \lambda T_{2}}, \\
b(\lambda) & =v_{2}\left(T_{2}\right) e^{-j \lambda T_{2}} .
\end{aligned}
$$

The NFT of a signal $q(t)$ consists of a continuous spectral function defined on the real axis $\lambda \in \mathbb{R}$

$$
\hat{q}(\lambda)=\frac{b(\lambda)}{a(\lambda)}, \quad \lambda \in \mathbb{R},
$$

and a discrete spectral function defined on the upper half complex plane $\mathbb{C}^{+}=\{\lambda: \Im(\lambda)>0\}$

$$
\tilde{q}\left(\lambda_{j}\right)=\frac{b\left(\lambda_{j}\right)}{\mathrm{d} a(\lambda) /\left.\mathrm{d} \lambda\right|_{\lambda=\lambda_{j}}}, j=1, \cdots, \mathbf{N},
$$

where $\lambda_{j}$ are eigenvalues and correspond to the (isolated) zeros of $a(\lambda)$ in $\mathbb{C}^{+}$, i.e., $a\left(\lambda_{j}\right)=0$.

From the discussions made, in order to compute the nonlinear spectrum of $q(t)$, the system of differential equations (5) needs to be solved in the interval $\left[T_{1}, T_{2}\right]$. Except for special cases, (5) needs to be solved numerically.

Numerical methods for the calculation of the forward nonlinear Fourier transform are divided into two classes in this article:

1) Methods which estimate the continuous spectrum by directly integrating the Zakharov-Shabat system; see Section IIII

2) Methods which find the (discrete) eigenvalues. Two approaches are suggested in this paper for this purpose. Similar to the continuous spectrum estimation, we can integrate the Zakharov-Shabat system numerically and obtain $a(\lambda)$. To find zeros of $a(\lambda)$, the scheme is often supplemented with a search method to locate eigenvalues in the upper half complex plane. One can also discretize and rewrite the Zakharov-Shabat eigenproblem $L v=\lambda v$ in the interval $\left[T_{1}, T_{2}\right]$ as a (large) matrix eigenvalue problem; see Section IV]

We begin by discussing methods which estimate the continuous spectrum.

\section{Numerical Methods for Computing the CONTINUOUS SPECTRUM}

In this section, we assume that $\lambda \in \mathbb{R}$ is given and provide algorithms for calculating the nonlinear Fourier coefficients $a(\lambda)$ and $b(\lambda)$. The continuous spectral function is then easily 
computed from (7). This process can be repeated to compute the spectral amplitudes for any desired finite set of continuous frequencies $\lambda$.

\section{A. Forward and Central Discretizations}

The most obvious method to attempt to solve (5) is the first-order Euler method or one of its variations [3].

Recall that the signal $q(t)$ is supported in the finite time interval $\left[T_{1}, T_{2}\right]$, and partition this interval uniformly according to the mesh $T_{1}<T_{1}+\epsilon<\cdots<T_{1}+N \epsilon=T_{2}$ with size $N$, i.e., with $\epsilon=\left(T_{2}-T_{1}\right) / N$. Let $q[k] \triangleq q\left(T_{1}+k \epsilon\right)$ and let

$$
P[k] \triangleq\left(\begin{array}{cc}
-j \lambda & q[k] \\
-q^{*}[k] & j \lambda
\end{array}\right) .
$$

Integrating both sides of (5) from $k \epsilon$ to $(k+1) \epsilon$ and assuming that the right hand side is constant over this interval, we get

$$
\begin{aligned}
v[k+1] & =A_{f}[k] v[k], \quad k=0, \ldots, N, \\
v[0] & =\left(\begin{array}{l}
1 \\
0
\end{array}\right) e^{-j \lambda T_{1}},
\end{aligned}
$$

where $A_{f}[k]=I_{2}+\epsilon P[k]$ and $I_{2}$ is the identity matrix. Equation (9) is iterated from $k=0$ to $k=N$ to find $v[N]$. The resulting vector is subsequently substituted in 6a - 6b to obtain $a(\lambda)$ and $b(\lambda)$.

We have implemented the Euler method for the calculation of the nonlinear Fourier transform of a number of pulse shapes. Unfortunately, the one-step Euler method does not produce satisfactory results for affordable small step sizes $\epsilon$.

One can improve upon the basic Euler method by considering the central-difference iteration [3],

$$
v[k+1]=v[k-1]+2 \epsilon P[k] v[k] .
$$

This makes the discretization second-order, i.e., the error $v\left(T_{1}+k \epsilon\right)-v[k]$ is of order $\mathcal{O}\left(\epsilon^{2}\right)$. Here an additional initial condition is required too, which can be obtained, e.g., by performing one step of the regular forward difference (9).

\section{B. Fourth-Order Runge-Kutta Method}

One can also employ higher-order integration schemes such as the Runge-Kutta methods. Improved results are obtained using the fourth-order Runge-Kutta method [4], [5]. However it takes significant time to estimate the spectrum using such higher-order numerical methods in real-time. Since the method, with its typical parameters, is quite slow and does not outperform some of the schemes suggested in the following sections, we do not elaborate on this method here; see [3] for details. However, for comparison purposes we will include this scheme in our numerical simulations given in Section VI.

\section{Layer-Peeling Method}

In Section IV. C of [Part I], we have calculated the nonlinear spectra of a rectangular pulse. One can approximate $q(t)$ as a piece-wise constant signal and use the layer-peeling property of the nonlinear Fourier transform to estimate the spectrum of any given signal. Let $a[k]$ and $b[k]$ be the nonlinear Fourier coefficients of $q(t)$ in the interval $\left[T_{1}, T_{1}+k \epsilon\right)$, and $x[k]$ and $y[k]$ coefficients in the small (rectangular) region $\left[T_{1}+k \epsilon, T_{1}+\right.$ $k \epsilon+\epsilon)$. The iterations of the layer-peeling method read

$$
\begin{aligned}
(a[k+1], b[k+1]) & =(a[k], b[k]) \circ(x[k], y[k]), \\
(a[0], b[0]) & =(1,0),
\end{aligned}
$$

where the operation is defined as in [1]

$$
\begin{aligned}
a[k+1] & =a[k] x[k]-b[k] \bar{y}[k], \\
b[k+1] & =a[k] y[k]+b[k] \bar{x}[k],
\end{aligned}
$$

in which

$$
\begin{aligned}
& x[k]=\left(\cos (D \epsilon)-j \frac{\lambda}{D} \sin (D \epsilon)\right) e^{j \lambda(t[k]-t[k-1])}, \\
& y[k]=\frac{-q^{*}[k]}{D} \sin (D \epsilon) e^{-j \lambda(t[k]+t[k-1])},
\end{aligned}
$$

and $\bar{x}[k](\lambda)=x^{*}[k]\left(\lambda^{*}\right), \quad \bar{y}[k](\lambda)=y^{*}[k]\left(\lambda^{*}\right), \quad D=$ $\sqrt{\lambda^{2}+|q[k]|^{2}}$. The desired coefficients are obtained as $a:=$ $a[N]$ and $b:=b[N]$. Note that the exponential factors in $x[k]$ and $y[k]$ enter (11) in a telescopic manner. As a result, for the numerical implementation, it is faster to drop these factors and just scale the resulting $a[N]$ and $b[N]$ coefficients by $\exp \left(j \lambda\left(T_{2}-T_{1}\right)\right)$ and $\exp \left(-j \lambda\left(T_{2}+T_{1}\right)\right)$, respectively. This, however, reduces the accuracy as it involves the product of large and small numbers.

We are motivated by [6] in which the layer-peeling identity (10) is mentioned as a property of the nonlinear Fourier transform. An equivalent presentation of this method is given in [7] as well.

Note further that a different numerical method, but with the same name (layer-peeling), exists in geophysics and fiber Bragg design [8]; however this method is not directly related to the forward NFT problem considered here.

We shall see in Section VI that the layer-peeling method gives remarkably accurate results in estimating the nonlinear Fourier transform.

\section{Crank-Nicolson Method}

In the Crank-Nicolson method, the derivative of the evolution parameter is approximated by a finite-difference approximation, e.g., forward discretization, and other functions are discretized by taking their average over the end points of the discretization interval:

$$
\frac{v[k+1]-v[k]}{\epsilon}=\frac{1}{2}(P[k] v[k]+P[k+1] v[k+1]),
$$

where $P[k]$ is defined in (8). This implicit iteration can be made explicit

$$
v[k+1]=A_{c n} v[k], \quad k=0, \cdots, N,
$$

with the initial condition (9), where

$$
A_{c n}=\left(I-\frac{\epsilon}{2} P[k+1]\right)^{-1}\left(I+\frac{\epsilon}{2} P[k]\right) .
$$

As we will see, this simple scheme too gives good results in estimating the nonlinear spectrum. 


\section{E. The Ablowitz-Ladik Discretization}

Ablowitz-Ladik (AL) discretization is an integrable discretization of the NLS equation in time domain [9]. In this section, we suggest using the Lax pairs of the Ablowitz-Ladik discretization of the NLS equation for solving the ZakharovShabat eigenproblem in the spectral domain.

Discretization sometimes breaks symmetries, making the discrete version of an integrable equation no longer integrable. A consequence of symmetry-breaking is that quantities which are conserved in the continuous model may no longer be invariant in the discrete model. A completely integrable Hamiltonian system with an infinite number of conserved quantities might have a discretized version with no, or few, conserved quantities. The discrete equation therefore does not quite mimic the essential features of the original equation if the step size is not small enough.

However, for some integrable equations, discretizations exist which are themselves completely integrable Hamiltonian systems, i.e., they possess an infinite number of conserved quantities and are linearizable by a Lax pair, and therefore are solvable by the nonlinear Fourier transform. Such developments exist for the NLS and Korteweg-de Vries (KdV) equations.

For the NLS equation, the integrable discrete version was introduced by Ablowitz and Ladik [9]. To illustrate the general idea, let us replace $1 \pm j \lambda \epsilon$ for small $\epsilon$ with $e^{ \pm j \lambda \epsilon}$ in the forward discretization method (9) (the opposite of what is usually done in practice). Let $z=e^{-j \lambda \epsilon}$ represent the discrete eigenvalue, $Q[k]=q[k] \epsilon$, and

$$
A_{a l}[k]=\left(\begin{array}{cc}
z & Q[k] \\
-Q^{*}[k] & z^{-1}
\end{array}\right) .
$$

The Ablowitz-Ladik iteration is

$$
v[k+1]=A_{a l}[k] v[k], \quad v[0]=\left(\begin{array}{l}
1 \\
0
\end{array}\right) e^{-j \lambda T_{1}} .
$$

Under the $z$ transformation, the upper half complex plane in $\lambda$ domain is mapped to the exterior of the unit circle in the $z$ domain. The continuous spectrum therefore lies on the unit circle $|z|=1$, while the discrete spectrum lies outside of the unit circle $|z|>1$.

One can rewrite the $R$-equation (12) in the eigenvalue form $L v[k]=z v[k]$, with the following $L$ operator

$$
L=\left(\begin{array}{cc}
\mathcal{Z} & -Q[k] \\
-Q^{*}[k-1] & \alpha[k-1] \mathcal{Z}^{-1}
\end{array}\right),
$$

where $\alpha[k]=1+|Q[k]|^{2}$, and $\mathcal{Z}$ is the shift operator, i.e., $\mathcal{Z}^{-1} x[k]=x[k-1], k \in \mathbb{Z}$. To the first order in $\epsilon, \alpha[k] \approx 1$ and (14) can be simplified to

$$
L=\left(\begin{array}{cc}
\mathcal{Z} & -Q[k] \\
-Q^{*}[k] & \mathcal{Z}^{-1}
\end{array}\right) .
$$

Given the $L$ operator (15), one can consider the $M$ operator of the continuous NLS equation and modify its elements such that the compatibility equation $L_{z}=[M, L]$ represents a discretized version of the NLS equation. It is not hard to verify that after doing so we are led to an $M$ operator resulting in the following discrete integrable NLS equation [9], [10]

$$
\begin{aligned}
j \frac{\mathrm{d} q[k]}{\mathrm{d} z}= & \frac{q[k+1]-2 q[k]+q[k-1]}{\epsilon^{2}} \\
& +|q[k]|^{2}(q[k+1]+q[k-1]) .
\end{aligned}
$$

Here the space derivative remains intact and the signal $q[k]$ is discretized in time, in such a way that the nonlinearity is somewhat averaged among three time samples. In the continuum limit $\epsilon \rightarrow 0$, (16) approaches the continuous NLS equation and its merits lie in the fact that it is integrable for any $\epsilon$, not just in the limit $\epsilon \rightarrow 0$. For example, soliton signals can be observed in this model for any $\epsilon$. The equation has its own infinite number constants of motion, approaching integrals of motion in the continuum limit. The operator $M$ which leads to (16), and the details of the nonlinear Fourier transform for (16) can be found in [9], [10].

We conclude that the Ablowitz-Ladik discretization can be used not only as a means to discretize the NLS equation in the time domain [11], but also as a means to solve the continuoustime Zakharov-Shabat system in the spectral domain. This is a non-finite-difference discretization, capable of dealing with oscillations $\exp ( \pm j \lambda t)$ in the Zakharov-Shabat system, which greatly enhances the accuracy of the one-step finite-difference methods.

Following Tao and Thiele's approach [6] and [4], we can also normalize the $A_{a l}[k]$ matrix

$$
v[k+1]=\frac{1}{\sqrt{1+|Q[k]|^{2}}}\left(\begin{array}{cc}
z & Q[k] \\
-Q[k]^{*} & z^{-1}
\end{array}\right) v[k] .
$$

The scale factor does not change the spectrum significantly, since it is canceled out in the ratios $\hat{q}=b / a$ and $\tilde{q}=b / a^{\prime}$, and also its effects are second-order in $\epsilon$. However, numerically, normalization may help in reducing the numerical error. In subsequent sections, we refer to (13) as the Ablowitz-Ladik method (AL1) and to (17) as the modified Ablowitz-Ladik method (AL2).

\section{Methods for Calculating the Discrete SPECTRUM}

In order to compute the discrete spectrum, the zeros of $a(\lambda)$ in the upper half complex plane must be found. One way to visualize this is to assume a two-dimensional mesh in $\mathbb{C}^{+}$and determine $a$ at all mesh points. Discrete eigenvalues are then easily identified by looking at the graph of $|a(\lambda)|$; in many cases they correspond to deep and narrow "wells" corresponding to the zeros of the magnitude of $a$.

As noted earlier, two types of methods are suggested to calculate the point spectrum.

1) One can use the integration-based algorithms mentioned in Section [II] which calculate nonlinear Fourier coefficients, and search for eigenvalues using a root finding method, such as the Newton-Raphson method. Such methods require good initial points and one needs to be careful about convergence [3].

2) It is also possible to rewrite the spectral problem for an operator as a (large) matrix eigenvalue problem. The 
point spectrum of the operator can be found in this way too.

\section{A. Discrete Spectrum via Search Methods}

To calculate the discrete spectral amplitude $\tilde{q}=b / a^{\prime}$, we require $\mathrm{d} a / \mathrm{d} \lambda$ as well. As we will show, information about the derivative of $a$ can be updated recursively along with the information about $a$, without resorting to approximate numerical differentiation.

Recall that the nonlinear Fourier coefficient $a(\lambda)$ is given by $6 a$

$$
a(\lambda)=v_{1}[N] e^{j \lambda t[N]} .
$$

Taking the derivative with respect to $\lambda$, we obtain

$$
\frac{\mathrm{d} a(\lambda)}{\mathrm{d} \lambda}=\left(\left(v_{1}[N]\right)^{\prime}+j t[N] v_{1}[N]\right) e^{j \lambda t[N]} .
$$

We can update the derivative information $\mathrm{d} v / \mathrm{d} \lambda$ along with $v$. In methods of Section III, the transformation of eigenvectors from $t[k]$ to $t[k+1]$ can be generally represented as

$$
v[k+1]=A[k] v[k],
$$

for some suitable one-step update matrix $A[k]$, which varies from method to method. Differentiating with respect to $\lambda$ and augmenting $v$ with $v^{\prime}=\mathrm{d} v / \mathrm{d} \lambda$, we get the iterations

$$
\begin{aligned}
v[k+1] & =A[k] v[k], \\
v^{\prime}[k+1] & =A^{\prime}[k] v[k]+A[k] v^{\prime}[k],
\end{aligned}
$$

with initial conditions

$$
v[0]=\left(\begin{array}{l}
1 \\
0
\end{array}\right) e^{-j \lambda t[0]}, \quad v^{\prime}[0]=\left(\begin{array}{c}
-j t[0] \\
0
\end{array}\right) e^{-j \lambda t[0]} .
$$

The derivative matrix $A^{\prime}$ depends on the method used.

For the forward discretization scheme:

$$
A_{f}^{\prime}=M_{1}=\left(\begin{array}{cc}
-j & 0 \\
0 & j
\end{array}\right) \epsilon \text {. }
$$

For the Crank-Nicolson scheme:

$$
A_{c n}^{\prime}=\frac{\epsilon}{2}\left(I-\frac{\epsilon}{2} P[k+1]\right)^{-1} \Sigma_{3}\left(I+A_{c n}[k]\right),
$$

where $\Sigma_{3}=\operatorname{diag}(-j, j)$.

For the Ablowitz-Ladik method:

$$
A_{a l}^{\prime}=\left(\begin{array}{cc}
-j z & 0 \\
0 & j z^{-1}
\end{array}\right) \epsilon .
$$

The desired coefficients are obtained at $k=N$ as follows

$$
\begin{aligned}
a(\lambda) & =v_{1}[N] e^{j \lambda t[N]}, \\
b(\lambda) & =v_{2}[N] e^{-j \lambda t[N]}, \\
a^{\prime}(\lambda) & =\left(v_{1}^{\prime}[N]+j t[N] v_{1}[N]\right) e^{j \lambda t[N]} .
\end{aligned}
$$

Similarly, the layer-peeling iteration can be augmented to update $a^{\prime}(\lambda)$ as well:

$$
\begin{aligned}
a^{\prime}[k+1] & =a^{\prime}[k] x[k]+a[k] x^{\prime}[k]-\left(b^{\prime}[k] \bar{y}[k]+b[k] \bar{y}^{\prime}[k]\right), \\
b^{\prime}[k+1] & =a^{\prime}[k] y[k]+a[k] y^{\prime}[k]+b^{\prime}[k] \bar{x}[k]+b[k] \bar{x}^{\prime}[k], \\
a^{\prime}[0] & =b^{\prime}[0]=0,
\end{aligned}
$$

where

$$
\begin{aligned}
x^{\prime}[k]= & j \epsilon\left(1-\frac{\lambda^{2}}{D^{2}}\right)\left(\cos (D \epsilon)-\frac{\sin (D \epsilon)}{D \epsilon}\right) e^{j \lambda \epsilon}, \\
y^{\prime}[k]= & -q^{*}[k]\left\{\frac{\lambda \epsilon}{D^{2}} \cos (D \epsilon)\right. \\
& \left.-\left(\frac{\lambda}{D^{3}}+j \frac{t[k]+t[k-1]}{D}\right) \sin (D \epsilon)\right\} e^{-j \lambda(t[k]+t[k-1])} .
\end{aligned}
$$

The expressions for $\bar{x}^{\prime}[k]$ and $\bar{y}^{\prime}[k]$ are similar, with all $j$ 's replaced with $-j$ and $q^{*}[k]$ replaced with $q[k]$.

With the derivative information being available, the NewtonRaphson method is a good scheme to search for the location of the (discrete) eigenvalues. The iteration for the complexvalued Newton-Raphson scheme is

$$
\lambda_{k+1}=\lambda_{k}-\alpha_{k} \frac{a\left(\lambda_{k}\right)}{a^{\prime}\left(\lambda_{k}\right)},
$$

where $\alpha_{k}$ is some step size modifier; usually $\alpha_{k}=1$. The iteration stops if $\lambda_{k}$ is almost stationary, i.e., if $\left|\alpha \frac{a}{a^{\prime}}\right|<\delta$ for a small $\delta$. In practice, the quadratic convergence of the scheme is often very fast and occurs in just a few iterations.

In data communications, since noise is usually small, the points in the transmitted constellation can (repeatedly) serve as the initial conditions for (19). In this case, convergence is usually achieved in a couple of iterations. For an unknown signal, random initial conditions are chosen. In either case, one or more sequence of Newton iterations have to be performed for any single eigenvalue.

To make sure that all of the eigenvalues are found, we can check the trace formula for $n=1,2,3$ [Part I]. The trace formula is a time frequency identity relating the hierarchy of infinitely many conserved quantities to the spectral components.

In general, the trace formula represents a time-domain conserved quantity as the sum of discrete and continuous spectral terms:

$$
E^{(k)}=E_{\mathrm{disc}}^{(k)}+E_{\mathrm{cont}}^{(k)}, \quad k=0,1, \cdots,
$$

where

$$
\begin{aligned}
& E_{\text {disc }}^{(k)}=\frac{4}{k} \sum_{i=1}^{\mathrm{N}} \Im\left(\lambda_{i}^{k}\right), \\
& E_{\text {cont }}^{(k)}=\frac{1}{\pi} \int_{-\infty}^{\infty} \lambda^{k-1} \log \left(1+|\hat{q}(\lambda)|^{2}\right) d \lambda,
\end{aligned}
$$

and where $E^{(k)}$ are time-domain conserved quantities (functionals of the signal). The first few conserved quantities are energy

$$
E^{(1)}=\int_{-\infty}^{\infty}|q(t, z)|^{2} d t
$$

momentum

$$
E^{(2)}=\frac{1}{2 j} \int_{-\infty}^{\infty} q(t) \frac{\mathrm{d} q^{*}(t)}{\mathrm{d} t} d t
$$


and Hamiltonian

$$
E^{(3)}=\frac{1}{(2 j)^{2}} \int_{-\infty}^{\infty}\left(|q(t)|^{4}-\left|\frac{\mathrm{d} q(t)}{\mathrm{d} t}\right|^{2}\right) \mathrm{d} t .
$$

For $n=1$, the trace formula is a kind of Parseval's identity (Plancherel's theorem), representing the total energy of the signal in time as the sum of the energy of the discrete and continuous spectral functions. When satisfied, the Parseval's identity ensures that all of the signal energy has been accounted for.

We first calculate the continuous spectrum and its "energy terms" for a sufficiently fine mesh on the real $\lambda$ axis. The norm of the error vector

$$
E_{\text {error }}=\left(E^{(1)}-E_{\text {cont }}^{(1)}, E^{(2)}-E_{\text {cont }}^{(2)}, E^{(3)}-E_{\text {cont }}^{(3)}\right),
$$

gives an estimate on the number of remaining eigenvalues. When a new eigenvalue $\lambda$ is found, this error is updated as

$$
E_{\text {error }}:=E_{\text {error }}-\left(4 \Im \lambda, 2 \Im \lambda^{2}, \frac{4}{3} \Im \lambda^{3}\right) .
$$

The process is repeated until $\left\|E_{\text {error }}\right\|$ is less than a small prescribed tolerance value.

To summarize, given the signal $q(t)$, its nonlinear Fourier transform can be computed based on Algorithm 1.

\section{B. Discrete Spectrum as a Matrix Eigenvalue Problem}

The methods mentioned in Section IV-A find the discrete spectrum by searching for eigenvalues in the upper half complex plane. Sometimes it is desirable to have all eigenvalues at once, which can be done by solving a matrix eigenvalue problem [5]. These schemes obviously estimate only (discrete) eigenvalues and do not give information on the rest of the spectrum. Since the matrix eigenvalue problem can be solved quickly for small-sized problems, it might take less computational effort to compute the discrete spectrum in this way. In addition, one does not already need the continuous spectrum to estimate the size of the discrete spectrum. On the other hand, for large matrices that arise when a large number of signal samples are used, the matrix eigenproblem (which is not Hermitian) is slow and it may be better to find the discrete spectrum using the search-based methods. The matrix-based methods also have the disadvantages that they can generate a large number of spurious eigenvalues, and one may not be able to restrict the algorithm for finding eigenvalues of a matrix to a certain region of the complex plane.

Below we rewrite some of the methods mentioned in the Section [III as a regular matrix eigenvalue problem, for the computation of the discrete spectrum.

1) Central-Difference Eigenproblem: The matrix eigenvalue problem can be formulated in the time domain or the frequency domain [5]. Consider the Zakharov-Shabat system in the form $L v=\lambda v$

$$
j\left(\begin{array}{cc}
\frac{\partial}{\partial t} & -q \\
-q^{*} & -\frac{\partial}{\partial t}
\end{array}\right) v=\lambda v .
$$

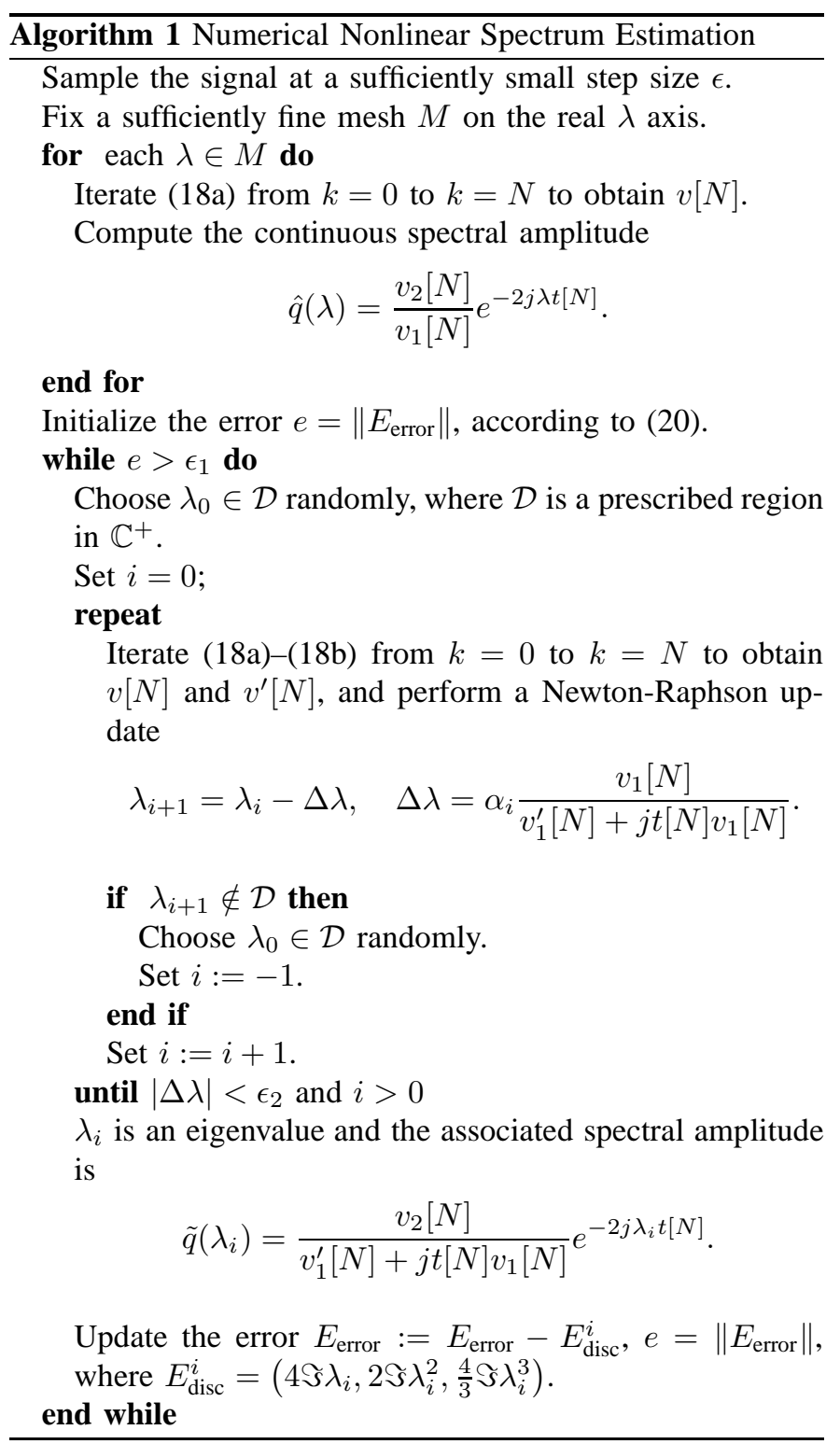

In the time domain, one can replace the time derivative $\partial / \partial t$ by the central finite-difference matrix

$$
D=\frac{1}{2 \epsilon}\left(\begin{array}{ccccc}
0 & 1 & 0 & \cdots & -1 \\
-1 & 0 & 1 & \cdots & 0 \\
& & & \cdots & \\
0 & 0 & -1 & 0 & 1 \\
1 & 0 & 0 & -1 & 0
\end{array}\right),
$$

and expand (21) as

$$
j\left(\begin{array}{cc}
D & -\operatorname{diag}(q[k]) \\
-\operatorname{diag}\left(q^{*}[k]\right) & -D
\end{array}\right) v[k]=\lambda v[k] .
$$

The point spectrum is contained in the eigenvalues of the matrix in the left hand side of (22).

Eigenvalues of a real symmetric or Hermitian matrix $A$ can be found relatively efficiently, owing to the existence of a complete orthonormal basis and the stability of the eigenvalues. In this case, a unitary matrix $P$ can be designed such that the sequence of similarity transformations $A_{k+1}=P^{T} A_{k} P$, $A_{0}=A$, converges to an almost diagonal (or a triangular) 
matrix. The similarity transformation can be found using, e.g., the QR factorization or the Householder transformation [12].

Unfortunately, most of the useful statements about computations using Hermitian matrices cannot usually be generalized to non-Hermitian matrices. As a result, the eigenvalues of a non-Hermitian matrix (corresponding to a nonself-adjoint operator) are markedly difficult to calculate [12], [13]. Running a general-purpose eigenvalue calculation routine on (22) may therefore not be the most efficient way to get eigenvalues $\left(\mathcal{O}\left(N^{3}\right)\right)$. One can study the general-purpose numerical eigenvalue algorithms in [12] and tailor them to the discrete Zakharov-Shabat spectral problems in this paper. Note that matrix $D$ is (almost) tridiagonal. Thus the eigenproblem $A x=\lambda x$ for (22), or equivalently discretization of (27) gives a second-order recursive equation $z_{n+2}=\alpha(\lambda) z_{n+1}+\beta(\lambda) z_{n}$. One can then obtain polynomial $a(\lambda):=z_{N}(\lambda)$ recursively in $\mathcal{O}\left(N^{2}\right)$ operations and proceed to find its roots. Alternatively, $\operatorname{det}(\lambda I-A)$ can be calculated recursively.

2) Ablowitz-Ladik Eigenproblem: We can also rewrite the Ablowitz-Ladik discretization as a matrix eigenvalue problem. Using the $L$ operator (14), we obtain

$$
\begin{aligned}
v_{1}[k+1]-Q[k] v_{2}[k] & =z v_{1}[k], \\
-Q^{*}[k-1] v_{1}[k]+\alpha[k-1] v_{2}[k-1] & =z v_{2}[k],
\end{aligned}
$$

which consequently takes the form

$$
\left(\begin{array}{cc}
U_{1} & -\operatorname{diag}(Q[k]) \\
-\operatorname{diag}\left(Q^{*}[k-1]\right) & U_{2}^{T}
\end{array}\right) v=z v,
$$

in which

$$
\begin{aligned}
U_{1} & =\left(\begin{array}{ccccc}
0 & 1 & 0 & \cdots & 0 \\
0 & 0 & 1 & \cdots & 0 \\
& & & \cdots & \\
0 & 0 & 0 & 0 & 1 \\
1 & 0 & 0 & 0 & 0
\end{array}\right), \\
U_{2} & =\left(\begin{array}{ccccc}
0 & \alpha[0] & 0 & \cdots & 0 \\
0 & 0 & \alpha[1] & \cdots & 0 \\
0 & 0 & 0 & 0 & \alpha[N-1] \\
\alpha[N] & 0 & 0 & 0 & 0
\end{array}\right),
\end{aligned}
$$

and $\operatorname{diag}(Q[k])=\operatorname{diag}(Q[0], \cdots, Q[N]), \operatorname{diag}\left(Q^{*}[k-1]\right)=$ $\operatorname{diag}\left(Q^{*}[N], Q^{*}[0] \cdots, Q^{*}[N-1]\right)$. Note that all shifting operations are cyclic, so that all vector indices $k$ remain in the interval $0 \leq k \leq N$.

Similar results can be obtained for the simplified operator (15) instead of (14). This corresponds to the above discretization with $U_{2}:=U_{1}$ and $-Q^{*}[k-1]$ replaced with $-Q^{*}[k]$. Similarly, one can rewrite the normalized Ablowitz-Ladik iteration (17) as a matrix eigenvalue problem. This corresponds to the $L$ operator (14) with the top left and bottom right entries replaced, respectively, with $\sqrt{\alpha[k]} \mathcal{Z}$ and $\sqrt{\alpha[k-1]} \mathcal{Z}^{-1}$. Accordingly $v_{1}[k+1]$ and $\alpha[k-1] v_{2}[k-1]$ in (23a) and (23b) are replaced, respectively, with $\sqrt{\alpha[k]} v_{1}[k+1]$ and $\sqrt{\alpha[k-1]} v_{2}[k+1]$ and $U_{1}:=U_{2}$ in the given expressions.

3) Spectral Method: In the frequency domain, one can approximate derivatives with the help of the Fourier transform.
Let us assume that

$$
v(t)=\sum_{k=-\frac{M}{2}}^{\frac{M}{2}}\left(\begin{array}{l}
\alpha_{k} \\
\beta_{k}
\end{array}\right) e^{\frac{j 2 k \pi t}{T}}, \quad q(t)=\sum_{k=-\frac{M}{2}}^{\frac{M}{2}} \gamma_{k} e^{\frac{j 2 k \pi t}{T}},
$$

where $T=T_{2}-T_{1}$ and $M+1$ is the maximum number of frequencies in all variables. Then the Zakharov-Shabat system is

$$
\begin{aligned}
-\alpha_{k} \frac{2 \pi k}{T}-j \sum_{m=-\frac{M}{2}}^{\frac{M}{2}} \gamma_{k-m} \beta_{m} & =\lambda \alpha_{k}, \\
-j \sum_{m=-\frac{M}{2}}^{\frac{M}{2}} \gamma_{-k+m}^{*} \alpha_{m}+\beta_{k} \frac{2 \pi k}{T} & =\lambda \beta_{k} .
\end{aligned}
$$

Thus we obtain

$$
\left(\begin{array}{cc}
\Omega & \Gamma \\
-\Gamma^{H} & -\Omega
\end{array}\right)\left(\begin{array}{l}
\alpha \\
\beta
\end{array}\right)=\lambda\left(\begin{array}{l}
\alpha \\
\beta
\end{array}\right)
$$

where $\alpha=\left[\alpha_{-\frac{M}{2}}, \ldots, \alpha_{\frac{M}{2}}\right]^{T}, \beta=\left[\beta_{-\frac{M}{2}}, \ldots, \beta_{\frac{M}{2}}\right]^{T}, \Omega=$ $-\frac{2 \pi}{T} \operatorname{diag}\left(-\frac{M}{2}, \ldots, \frac{M}{2}\right)$ and $\Gamma$ is a $(M+1)^{2} \times(M+1)$ Toeplitz matrix with the first row

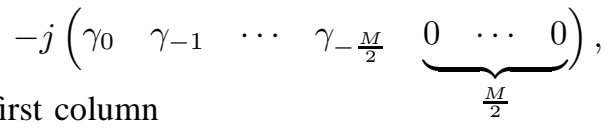

and the first column

$$
-j\left(\begin{array}{llllll}
\gamma_{0} & \gamma_{1} & \cdots & \gamma_{\frac{M}{2}} & \underbrace{\begin{array}{lll}
0 & \cdots & 0
\end{array}}_{\frac{M}{2}})^{T} .
\end{array}\right.
$$

The point spectrum is thus found by looking at the eigenvalues of the matrix

$$
A=\left(\begin{array}{cc}
\Omega & \Gamma \\
-\Gamma^{H} & -\Omega
\end{array}\right) .
$$

\section{Running Time, Convergence and Stability of THE NUMERICAL METHODS}

The numerical methods discussed in this paper are firstorder matrix iterations and therefore the running time of all of them to get $v[N]$ is $\mathcal{O}(N)$ multiplications and additions per eigenvalue. This corresponds to a complexity of $\mathcal{O}\left(N^{2}\right)$ operations for the calculation of the continuous spectrum on a mesh with $N$ eigenvalues. The exact number of operations depends on the details of the implementation and the memory requirement of the method. All iterative methods thus take about the same time asymptotically, albeit with different coefficients.

An important observation is that, while the Fast Fourier Transform (FFT) takes $\mathcal{O}\left(N \log _{2} N\right)$ operations to calculate the spectral amplitudes of a vector with length $N$ at $N$ equispaced frequencies, the complexity of the methods described in this paper to compute the continuous spectrum are $\mathcal{O}\left(N^{2}\right)$. Similarly, it takes $\mathcal{O}(\mathrm{N} N)$ operations to calculate the discrete spectrum. In other words, so far we do not exploit the potentially repetitive operations in our computations.

It is evident from (6a) that as $T_{2} \rightarrow \infty, v_{1}[k]$ should grow as $\sim \exp \left(-j \lambda T_{2}\right)$ so that $a(\lambda)$ is a finite complex number. The canonical eigenvector $v\left[k ; T_{1}, T_{2}\right]$ thus has an unbounded 
component as $T_{2} \rightarrow \infty$ (i.e., $\|v[k]\| \rightarrow \infty$ ). One can, however, normalize $v_{1}$ and $v_{2}$ according to

$$
\begin{aligned}
& u_{1}=v_{1} e^{j \lambda t}, \\
& u_{2}=v_{2} e^{-j \lambda t},
\end{aligned}
$$

and transform (5) to

$$
u_{t}=\left(\begin{array}{cc}
0 & q(t) e^{2 j \lambda t} \\
-q^{*}(t) e^{-2 j \lambda t} & 0
\end{array}\right) u, u\left(T_{1}, \lambda\right)=\left(\begin{array}{l}
1 \\
0
\end{array}\right) .
$$

The desired coefficients are simply $a(\lambda)=u_{1}\left(T_{2}\right)$ and $b(\lambda)=u_{2}\left(T_{2}\right)$. Consequently, if one is interested in obtaining eigenvectors $v[k]$ in addition to the coefficients $a(\lambda)$ and $b(\lambda)$, the discretization of the normalized system (24) has better numerical properties:

$$
\begin{aligned}
\left(\begin{array}{c}
a[k+1] \\
b[k+1]
\end{array}\right) & =\left(\begin{array}{cc}
1 & Q[k] z^{-2 k} \\
-Q^{*}[k] z^{2 k} & 1
\end{array}\right)\left(\begin{array}{l}
a[k] \\
b[k]
\end{array}\right), \\
\left(\begin{array}{l}
a[0] \\
b[0]
\end{array}\right) & =\left(\begin{array}{l}
1 \\
0
\end{array}\right) .
\end{aligned}
$$

The nonlinear Fourier coefficients are obtained as $a:=a[N]$ and $b:=b[N]$. The discrete nonlinear Fourier transform mentioned in [6] is thus the forward discretization of the normalized Zakharov-Shabat system (24).

We are interested in the convergence of $v[k]$ (or $a(\lambda)$ and $b(\lambda))$ as a function of $N$ for fixed values of $T_{1}$ and $T_{2}$. That is to say, we require that the error $e=\left\|v\left(T_{1}+k \epsilon\right)-v[k]\right\| \rightarrow 0$ as $N \rightarrow \infty$ (for fixed $T_{1}$ and $T_{2}$ ). The (global) error in all methods described in this paper is at least $\mathcal{O}(\epsilon)$, and therefore all these methods are convergent.

Some of these methods are, however, not stable. This is partially because the Zakharov-Shabat system can have unbounded solutions for $\lambda \in \mathbb{C}^{+}$, i.e., $\|v(t)\| \rightarrow \infty$ as $t \rightarrow \infty$. Errors can potentially be amplified by the system unstable dynamics. One should be cautious about the normalized system (24) as well. For example, forward discretization of the normalized system (25) gives a first-order iteration $x[k+1]=A_{f n}[k] x[k]$. The eigenvalues of the matrix $A_{f n}[k]$ in this method are

$$
s_{1,2}=1 \pm j \epsilon|q[k]| .
$$

It follows that the forward discretization of (24) gives rise to eigenvalues outside of the unit disk, $|s|>1$. As a result, first-order discretization of (24) are also unstable. In cases where $|s|>1$, we can consider normalizing the iterations by dividing $A_{f n}[k]$ by $\sqrt{\operatorname{det} A_{f n}[k]}$ (in the case of (25), dividing the right-hand side by $\sqrt{1+|Q[k]|^{2}}$ ). The resulting iteration has eigenvalues inside the unit disk. For $\epsilon \ll 1$ the effect is only second order in $\epsilon$, however it helps in managing the numerical error if larger values of $\epsilon$ are chosen.

An issue pertinent to numerical methods is chaos. Chaos and numerical instability of finite-difference discretizations has been observed in [14] for the sine-Gordon equation, which is also integrable and shares a number of basic properties with the NLS equation. In [15], the authors conclude that the standard discretizations of the cubic nonlinear Schrödinger equation may lead to spurious numerical behavior. This instability is related to the homoclinic orbits of the NLS equation, i.e., it occurs if the initial signal $q(t, 0)$ is chosen to be close to the homoclinic orbit of the equation. It disappears only if the step size is made sufficiently small, which can be smaller than what is desired in practice.

It is shown in [15] that the Ablowitz-Ladik discretization of the NLS equation (in time) has the desirable property that chaos and numerical instability, which are sometimes present in finite-difference discretizations of the NLS equation, do not appear at all. Though these results are for the original time-domain equations, the issue can occur in the spectral eigenvalue problem (5) as well, if the signal $q[k]$ is close to a certain family of functions (related to $\sin \omega t$ and $\cos \omega t$ ). Therefore, among discretizations studied, the Ablowitz-Ladik discretization of the Zakharov-Shabat system is immune to chaos and numerical instability. This is particularly important in the presence of amplifier noise, where chaos can be more problematic.

\section{Testing AND COMPARING the Numerical METHODS}

In this section, we test and compare the ability of the suggested numerical schemes to estimate the nonlinear Fourier transform (with respect to the Zakharov-Shabat system) of various signals. Numerical results are compared against analytical formulae, in a few cases where such expressions exist. Our aim is to compare the speed and the precision of these schemes for various pulse shapes in order to determine which ones are best suited for subsequent simulation studies.

To derive the analytical formulae, recall that the continuous spectral function can be written as $\hat{q}(\lambda)=\lim _{t \rightarrow \infty} y(t, \lambda)$, in which $y(t, \lambda)$ satisfies [1]

$$
\left\{\begin{array}{l}
\frac{\mathrm{d} y(t, \lambda)}{\mathrm{d} t}+q(t) e^{2 j \lambda t} y^{2}(t, \lambda)+q^{*}(t) e^{-2 j \lambda t}=0, \\
y(-\infty, \lambda)=0
\end{array}\right.
$$

Similarly, one can solve the second-order differential equation

$$
\left\{\begin{array}{l}
\frac{\mathrm{d}^{2} z(t, \lambda)}{\mathrm{d} t^{2}}-\left(2 j \lambda+\frac{q_{t}}{q}\right) \frac{\mathrm{d} z(t, \lambda)}{\mathrm{d} t}+|q|^{2} z(t, \lambda)=0, \\
z(-\infty, \lambda)=1, \quad \frac{\mathrm{d} z(-\infty, \lambda)}{\mathrm{d} t}=0,
\end{array}\right.
$$

and obtain $a(\lambda)=\lim _{t \rightarrow \infty} z(t, \lambda)$. The zeros of $a(\lambda)$ form the discrete spectrum.

In the following, the discrete spectrum is found and compared using the following matrix-based schemes:

1) central-difference method;

2) spectral method;

3) Ablowitz-Ladik discretization with no normalization (AL1);

4) Ablowitz-Ladik discretization with normalization (AL2). In the matrix-based schemes, the entire point spectrum is found at once by solving a matrix eigenvalue problem.

The complete spectrum is found using search-based methods:

1) forward discretization method;

2) fourth-order Runge-Kutta scheme;

3) layer-peeling methods; 

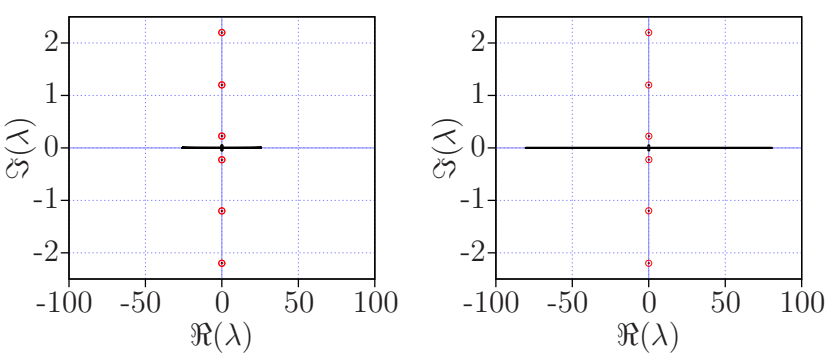

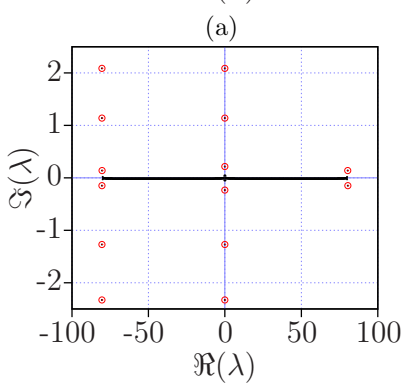

(c)

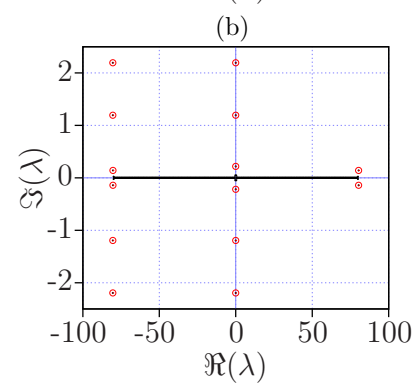

(d)
Fig. 1. Discrete spectrum of the Satsuma-Yajima signal with $A=2.7$ using (a) central-difference method, (b) spectral method, (c) Ablowitz-Ladik scheme, (d) modified Ablowitz-Ladik scheme.

4) Crank-Nicolson method;

5) AL discretization;

6) AL discretization with normalization.

In search-based methods, the Newton method is used together with the trace formula to find both discrete and continuous spectra.

Each of the following signals is sampled uniformly using a total of $n$ samples in a time window containing $99 \%$ of the signal energy.

\section{A. Satsuma-Yajima Signals}

One signal with known spectrum is the Satsuma-Yajima function [16]

$$
q(t)=A \operatorname{sech}(t), \quad A \geq 0 .
$$

Solving the initial value problem (5) (or 26) analytically, the following continuous spectral function is obtained [16]

$$
\hat{q}(\lambda)=-\frac{\Gamma\left(-j \lambda+\frac{1}{2}+A\right) \Gamma\left(-j \lambda+\frac{1}{2}-A\right)}{\Gamma^{2}\left(-j \lambda+\frac{1}{2}\right)} \frac{\sin (\pi A)}{\cosh (\pi \lambda)} .
$$

The discrete spectrum is the set of zeros of $a(\lambda)$, i.e., poles of $\hat{q}(\lambda)$ (when analytically extended in $\mathbb{C}^{+}$). Recalling that $\Gamma(x)$ has no zeros and is unbounded for $x=0,-1,-2, \ldots$, it follows that the discrete spectrum consists of $\mathrm{N}=\left\lfloor A+\frac{1}{2}-\epsilon\right\rfloor$ eigenvalues

$$
\lambda=\left\{\left(A-\frac{1}{2}\right) j,\left(A-\frac{3}{2}\right) j, \cdots\right\}, \quad \Im(\lambda)>0 .
$$

In the special case in which $A$ is an integer, $A=\mathrm{N}$, the Satsuma-Yajima signal is a pure $\mathrm{N}$-soliton with $\mathrm{N}$ eigenvalues, and the continuous spectral function is zero.

Figs. 11 2 and 3 give the numerical results for $A=2.7$, $N=2^{10}$. The value of $A$ is chosen so that both the discrete and continuous spectrum are present.

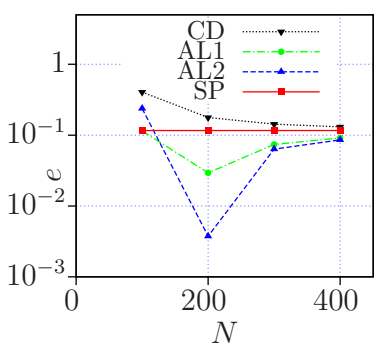

(a)

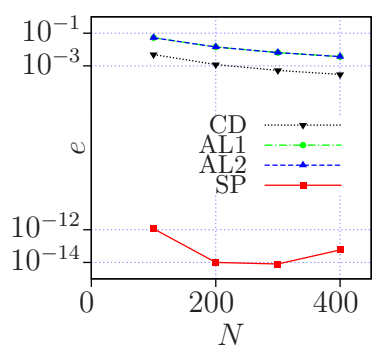

(b)
Fig. 2. Error in estimating (a) the smallest eigenvalue and (b) the largest eigenvalue of the Satsuma-Yajima signal $q(t)=2.7 \operatorname{sech}(t)$ as a function of the number of sample points $N$ using matrix eigenvalue methods. The Ablowitz-Ladik method 1 is the method of Section IV-B2 with no normalization, and the Ablowitz-Ladik method 2 is the same scheme with normalization.

Fig. 1 shows that, in this example, the spectral and centraldifference methods produce good results among the matrixbased methods in estimating the discrete eigenvalues $\lambda=$ $0.2 j, 1.2 j, 2.2 j$. All methods generate a large number of spurious eigenvalues along the real axis. This behavior might be viewed as a tendency of the algorithms to generate the continuous spectrum too. However the spurious eigenvalues do not disappear completely even when the continuous spectrum is absent (when $A$ is an integer); only their range becomes more limited. The spurious eigenvalues across the real axis can easily be filtered, since their imaginary part has negligible amplitude. The AL methods, with and without normalization, produce the same eigenvalues plus another vertical line of spurious eigenvalues having a large negative real part. Normalization in the AL scheme does not make a significant difference in this example.

Fig. 2 shows the accuracy of the various matrix-based methods in estimating the smallest and largest eigenvalues of $q=2.7 \operatorname{sech}(t)$ in terms of the number of the sample points $N$. As the number of sample points $N$ is decreased, the spectral and central-difference methods maintain reasonable precisions, while the accuracy of the AL schemes quickly deteriorates. One can check that in these cases, the error in the approximation $e^{j \lambda \epsilon} \approx 1+j \lambda \epsilon$ becomes large (since $\epsilon \gg 1$ ).

It can be seen that the relative performance of the numerical methods depends on the eigenvalue and the number of signal samples $N$. The spectral method is generally more accurate than the other matrix-based methods. The AL discretizations seem to perform well as long as $\lambda \epsilon \ll 1$, i.e., when estimating eigenvalues with small size or when $N \geq 200$. The $\mathrm{AL}$ discretization eventually breaks down at about $N=50$ as the analogy between the continuous and discrete NLS equation is no longer justified at such low resolutions, whereas other schemes continue to track the eigenvalues to some accuracy. In other words, what the AL methods find at such small values of $N$ is the spectrum of the discrete soliton-bearing NLS equation, which is not a feature of finite-difference discretizations. (In fact, it is essential for this algorithm to deviate from the finite-difference discretizations as $N$ is reduced, to produce appropriate solitons with few samples.) The running time of all matrix eigenvalue methods is about the same.

Search-based methods can be used to estimate the point 


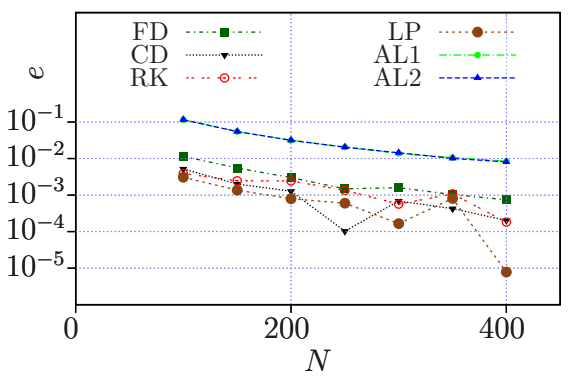

Fig. 3. Error in estimating the largest eigenvalue of Satsuma-Yajima signal $q(t)=2.7 \operatorname{sech}(t)$ as a function of the number of sample points $N$ using search-based methods.

spectrum as well. Here we use the Newton method with random initial points to locate eigenvalues in $\mathbb{C}^{+}$. Naturally, we limit ourselves to a rectangular region in the complex plan, slightly above the real axis to avoid potential spurious eigenvalues. Since the number of eigenvalues is not known a priori, the continuous spectrum is found first so as to give an estimate of the energy of the discrete spectrum. It is essential that the continuous spectrum is estimated accurately so that a good estimate of the energy of the discrete spectrum can be obtained. Once this energy is known, and a suitable (rectangular) search region in $\mathbb{C}^{+}$is chosen, the Newton method is often able to locate all of the discrete eigenvalues using just a few iterations.

Fig. 3 shows the accuracy of the searched-based methods in estimating the largest eigenvalue of the signal $q(t)=2.7 \operatorname{sech}(t)$. The Runge-Kutta, layer-peeling and CrankNicolson methods have about the same accuracy, followed closely by forward discretization. Since this is the largest eigenvalue, the AL schemes are not quite as accurate. As noted above, comparison at smaller values of $N$ is not illustrative, as the AL estimate quickly deviates from $\lambda_{\max }$ of the continuous signal.

The Runge-Kutta method, at the accuracies shown in the above graphs, is of course very slow, and is not a practical method to implement. The running time for the other schemes is approximately the same. Search-based methods take an order of magnitude more time than matrix-based methods when $N$ is small. These methods fail when $N$ becomes too small $(N<200)$, since the large error in estimating the energy terms of the continuous spectrum negatively influences the stopping criteria and consequently degrades the Newton increments. For large $N$, on the other hand, the $\mathrm{QR}$ factorization, which takes $\mathcal{O}\left(N^{3}\right)$ operations in calculating the eigenvalues of a matrix, becomes quite slow and restricts the use of matrixbased methods.

The same conclusions are observed for various choices of real or complex parameter $A$. As $|A|$ is increased, as before, the spectral and finite-difference schemes produce the correct eigenvalues, and the AL methods generate the same eigenvalues plus an additional vertical strip of spurious eigenvalues. The range of the spurious eigenvalues across the real axis remains about the same. As the phase of $A$ is increased, the true (non-spurious) eigenvalues remain the same in all methods (as expected analytically), while some of the vertical
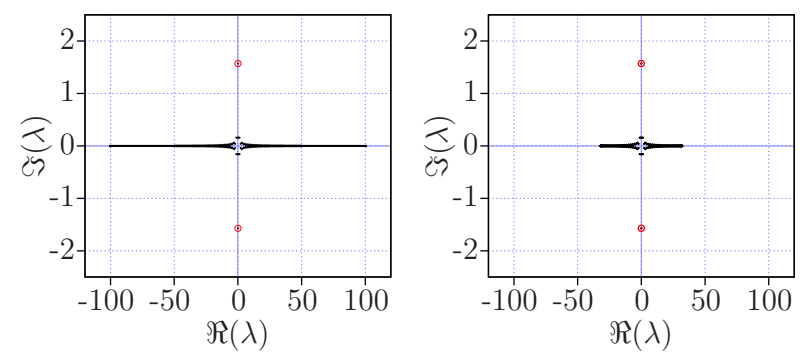

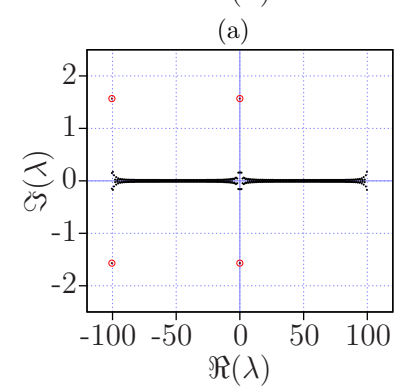

(c)

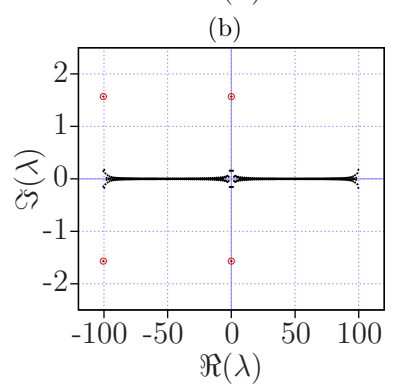

(d)
Fig. 4. Discrete spectrum of the rectangular pulse 28 with $A=2$, $T_{2}=-T_{1}=1$ using (a) spectral method, (b) central-difference method, (c) Ablowitz-Ladik scheme, (d) modified Ablowitz-Ladik scheme.

spurious eigenvalues in the $\mathrm{AL}$ schemes move from left to right or vice versa. The spectral and finite-difference schemes are relatively immune to these additional spurious eigenvalues. Normalization of the AL method sometimes produces slightly fewer spurious eigenvalues across the real axis, as can be seen in Figs. 1(c)-(d).

\section{B. Rectangular Pulse}

Consider the rectangular pulse

$$
q(t)= \begin{cases}A, & t \in\left[T_{1}, T_{2}\right] \\ 0, & \text { otherwise }\end{cases}
$$

It can be shown that the continuous spectrum is given by [1]

$$
\hat{q}(\lambda)=\frac{A^{*}}{j \lambda} e^{-2 j \lambda T_{2}}\left(1-\frac{D}{j \lambda} \cot \left(D\left(T_{2}-T_{1}\right)\right)\right)^{-1},
$$

where $D=\sqrt{\lambda^{2}+|A|^{2}}$. To calculate the discrete spectrum, the equation (27) is reduced to a simple constant coefficient second-order ordinary differential equation

$$
\frac{\mathrm{d}^{2} z}{\mathrm{~d} t^{2}}-2 j \lambda \frac{\mathrm{d} z}{\mathrm{~d} t}+|A|^{2} z=0, \quad z\left(T_{1}\right)=1, z^{\prime}\left(T_{1}\right)=0 .
$$

It is easy to verify that the eigenvalues are the solutions of

$$
e^{2 j\left(T_{2}-T_{1}\right) \sqrt{\lambda^{2}+|A|^{2}}}=\frac{\lambda+\sqrt{\lambda^{2}+|A|^{2}}}{\lambda-\sqrt{\lambda^{2}+|A|^{2}}} .
$$

Following the causality and the layer-peeling property of the NFT, one can generalize the above result to piece-wise constant pulses. This is the basis of the layer-peeling method of Section $\amalg I-C$.

Figs. 4(a)-(d) show the results of numerically computing the discrete spectrum of a rectangular pulse with parameters $A=2, T_{2}=-T_{1}=1$, denoted hereafter by $q(t)=$ $2 \operatorname{rect}(t)$. The exact eigenvalue is found to be $\lambda=1.5713 j$, 


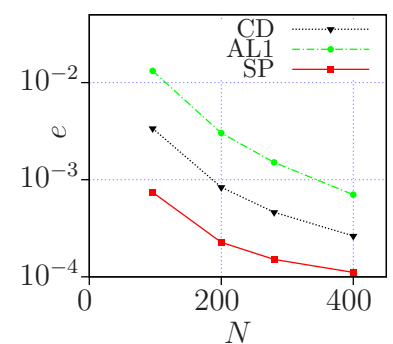

(a)

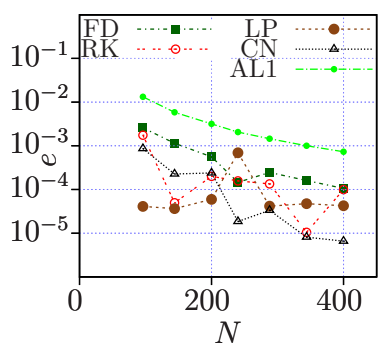

(b)
Fig. 5. Error in estimating the largest eigenvalue of the rectangular pulse $q(t)=2 \operatorname{rect}(t)$ as a function of the number of sample points $N$ using (a) matrix-based methods and (b) search-based methods.

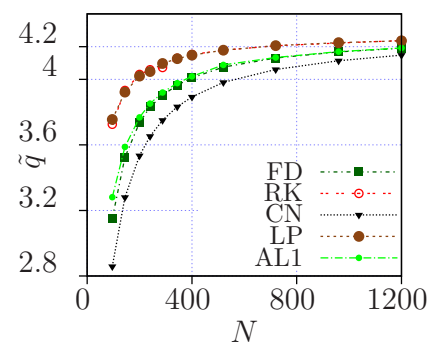

(a)

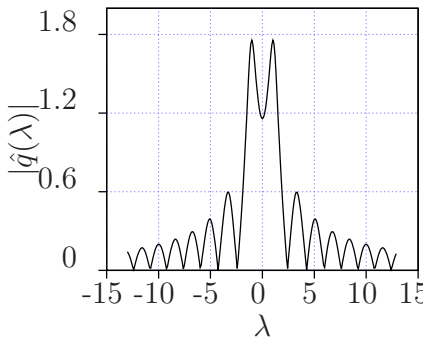

(b)
Fig. 6. (a) Convergence of the discrete spectral amplitude for the rectangular pulse $q(t)=2 \operatorname{rect}(t)$ as a function of the number of sample points $N$. Factor $-j$ is not shown in the figure. (b) Continuous spectrum.

by numerically finding the roots of 29) using the NewtonRaphson method. No other eigenvalue is found under a large number of random initial conditions. All methods generate the desired eigenvalue together with a large number of spurious eigenvalues across the real axis. The central-difference scheme visibly generates fewer spurious eigenvalues. The AblowitzLadik schemes produce two more eigenvalues with a large negative real part.

Fig. 5 compares the precision of various methods in estimating the nonlinear spectrum of $q(t)=2 \operatorname{rect}(t)$. The modified AL scheme performed the same as the basic AL scheme, and hence we do not include the modified AL scheme in the graphs.

Convergence to the discrete spectral amplitudes, if it occurs at all, is generally very slow compared with the convergence of eigenvalues. Fig. 6(a) shows the precision of various methods in estimating the discrete spectral amplitude of $q(t)=$

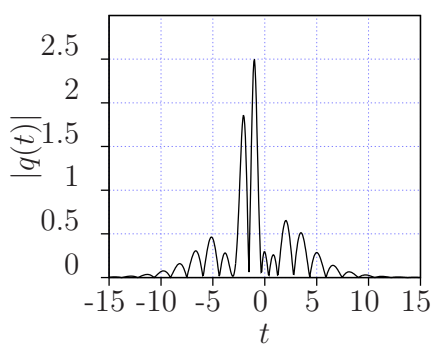

(a)

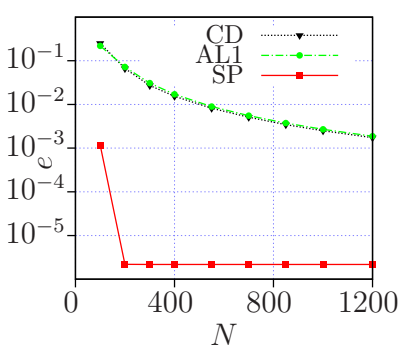

(b)
Fig. 7. (a) Amplitude profile of the 4-soliton signal with spectrum 30. (b) Error in estimating the eigenvalue $\lambda=1+0.5 j$.
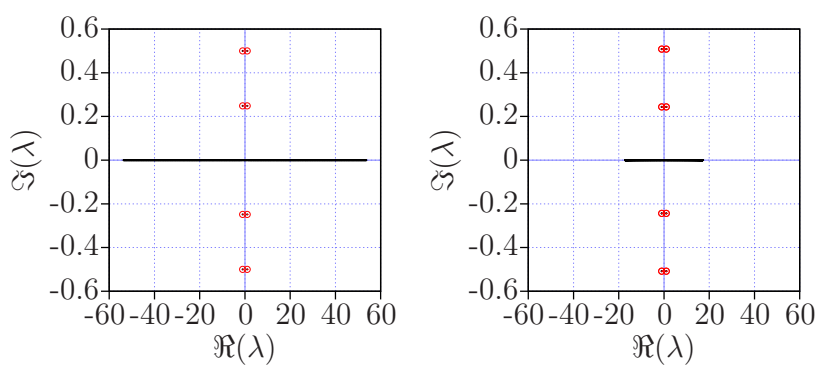

(a)

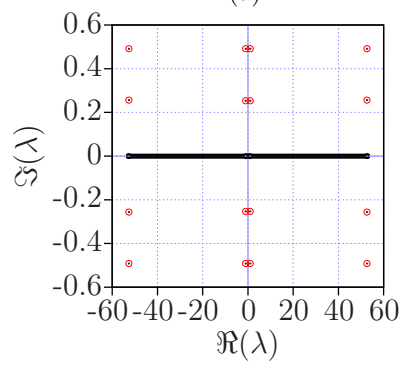

(c)

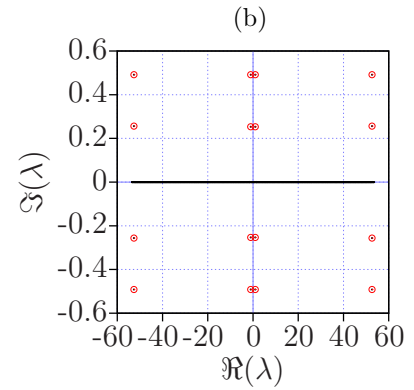

(d)

Fig. 8. Discrete spectrum of the 4-soliton signal with spectrum 30] using (a) Fourier method, (b) central-difference method, (c) Ablowitz-Ladik scheme, (d) modified Ablowitz-Ladik scheme.

$2 \operatorname{rect}(t)$. It can be seen that convergence does not occur until $N>1000$. Fig. 6(b) shows the continuous spectrum for the same function. All methods produced essentially the same continuous spectrum, except for some very slight variations near zero frequency.

As $|A|$ is increased, more eigenvalues appear on the imaginary axis. The distance between these eigenvalues becomes smaller as $|\lambda|$ is increased. All methods produce similar results, with the Ablowitz-Ladik methods reproducing the purely imaginary eigenvalues at spurious locations with large real part. Phase addition has no influence on any of these methods, as expected analytically.

\section{N-Soliton Signals}

We consider a 4-soliton signal with discrete spectrum

$$
\begin{array}{rlrl}
\tilde{q}(-1+0.25 j) & =1, & \tilde{q}(1+0.25 j) & =-j, \\
\tilde{q}(-1+0.5 j) & =-1, \quad \tilde{q}(1+0.5 j)=j .
\end{array}
$$

The 4-soliton is generated by solving the Riemann-Hilbert linear system of equations with zero continuous spectrum [1] and can be seen in Fig. 7(a). Figs. 8(a)-(d) show the discrete spectrum of the signal using various matrix-based methods. The relative accuracy of these schemes in estimating the eigenvalue $\lambda=1+0.5 j$ is shown in Fig. 7 (b). A very similar graph is obtained for other eigenvalues. Iterative methods fare similarly and their performance is shown in Figs. 9(a)-(b).

The convergence of the discrete spectral amplitudes $\tilde{q}\left(\lambda_{j}\right)$ is not quite satisfactory (Fig. 9(b)). Discrete spectral amplitudes associated with eigenvalues with small $\left|\Im\left(\lambda_{j}\right)\right|$ can be obtained with reasonable accuracy, although the convergence of $\tilde{q}\left(\lambda_{j}\right)$ is slower than the convergence of the eigenvalues themselves. On the other hand, discrete spectral amplitudes associated with eigenvalues with large $|\Im(\lambda)|$ are extremely sensitive 


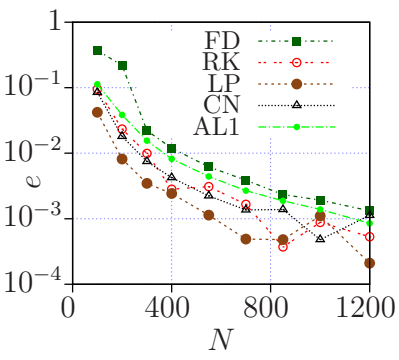

(a)

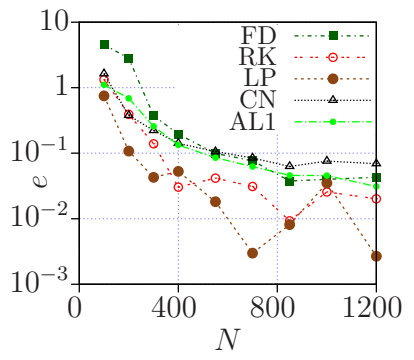

(b)
Fig. 9. (a) Error in estimating the eigenvalue $\lambda=-1+0.25 j$ in a 4-soliton using search-based methods. (b) Error in estimating the discrete spectral amplitude $|\tilde{q}|=1$.

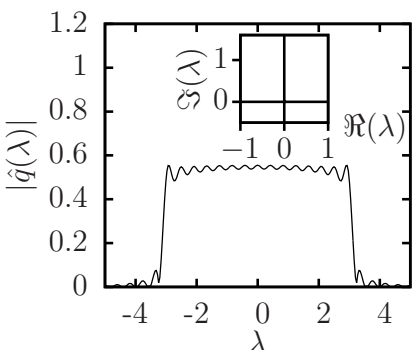

(a)

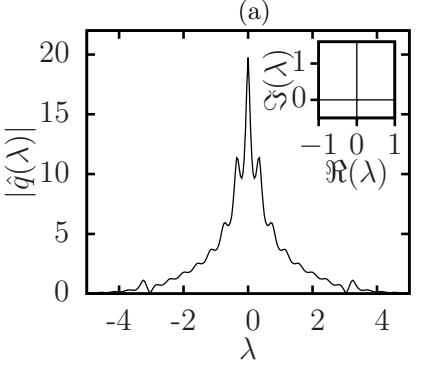

(c)

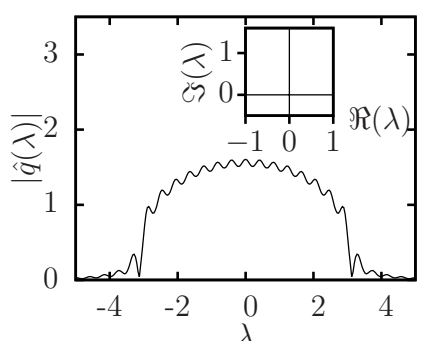

(b)

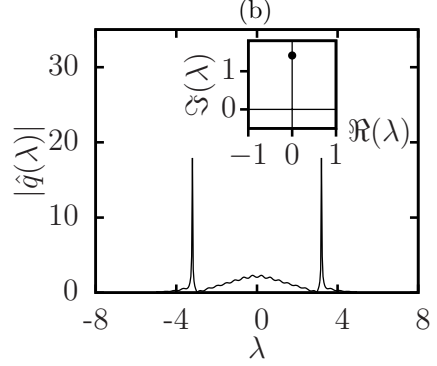

(d)
Fig. 10. Nonlinear Fourier transform of a sinc function with amplitude a) $A=1$, b) $A=2$, c) $A=3$, d) $A=4$.

to the location of eigenvalues and even slight changes in eigenvalues lead to radically different estimates for the spectral amplitudes. In fact, as the energy of the signal is increased by having eigenvalues with large $|\Im(\lambda)|$, the Riemann-Hilbert system becomes ill-conditioned. Therefore the discrete spectral amplitudes cannot generally be obtained using the methods discussed in this paper. It is illustrative to see the surface of $|a(\lambda)|$ in Fig. 21 The eigenvalues sometimes correspond to deep and narrow wells in the surface of $|a(\lambda)|$, and sometimes they correspond to flat minima. In cases that they correspond to narrow wells, the derivative $a^{\prime}(\lambda)$ is sensitive to the location of eigenvalues, leading to sensitivities in $\tilde{q}\left(\lambda_{j}\right)$.

Note that $\tilde{q}\left(\lambda_{j}\right)$ do not appear in the trace formula, and in particular they do not contribute to the signal energy. This part of the NFT controls the time center of the signal and influences the signal phase and shape too. Due to dependency on the time center of the signal and the fact that time center can hardly be used for digital transmission, the values of $\left|\tilde{q}\left(\lambda_{j}\right)\right|$ in the Riemann-Hilbert approach appear to be numerically chaotic and cannot carry much information. For this reason, we do not discuss these quantities in detail.

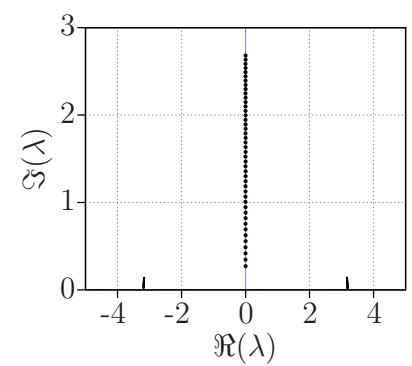

(a)

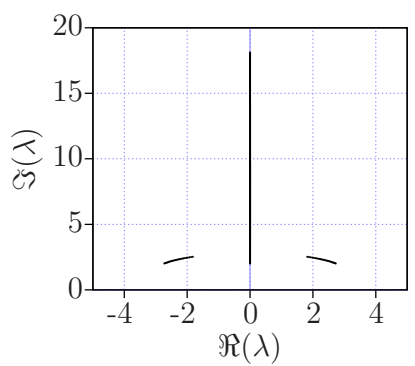

(b)
Fig. 11. Locus of eigenvalues of the sinc function under amplitude modulation: (a) $A=0$ to $A=5$, (b) $A=0$ to $A=20$.

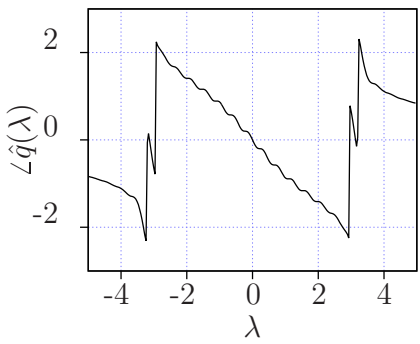

(a)

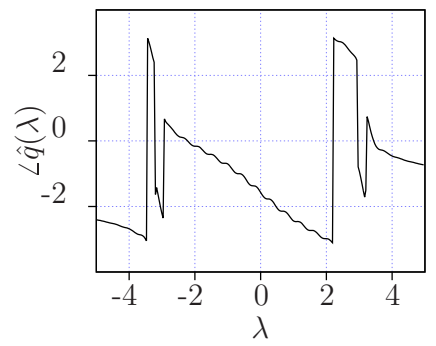

(b)
Fig. 12. Phase of the continuous spectrum of a sinc function when: (a) $A=4$ (b) $A=4 j$.

\section{NONLINEAR Fourier TRANSFORM OF Signals IN DATA COMMUNICATIONS}

In this section, we use the numerical methods discussed in Section II to compute the nonlinear Fourier transform of signals typically used in optical fiber transmission. The emphasis is on sinc functions as they constitute signal degreesof-freedom, but we also consider raised-cosine, $\operatorname{sech}(\omega t)$ and Gaussian signals. In particular, we study the effect of the amplitude and phase modulation on the structure of the nonlinear spectra. We will also discuss the spectrum of wavetrains formed by sinc functions.

Since the layer-peeling and the spectral methods give accurate results in estimating the nonlinear spectra, they are chosen for subsequent simulations.

\section{A. Spectra of the Nyquist Functions}

1) Amplitude and Phase Modulation of Sinc Functions: Fig. 10] shows the spectrum of $q(t)=A \operatorname{sinc}(2 t)$ under the amplitude modulation. Note that this function does not satisfy the Assumption 1 (a) since $q(t) \notin L^{1}(\mathbb{R})$. We therefore assume that the signal is truncated in a sufficiently large finite time interval. It can be seen that the nonlinear Fourier transform of $q(t)$ is all dispersive as $A$ is increased from zero, until about $A=\pi$ where a new eigenvalue emerges from the origin. Starting from $A=0$, the continuous spectrum is a rectangle, resembling the ordinary Fourier transform $-\mathcal{F}\left(q^{*}(t)\right)(2 \lambda)$. As $A$ is increased, the continuous spectral function is narrowed until $A=\pi$, where it looks like a delta function and its energy starts to deviate from the energy of the timedomain signal. As $A>\pi$ is further increased, the dominant eigenvalue on the $j \omega$ axis moves up until $A=1.27 \pi$, where 


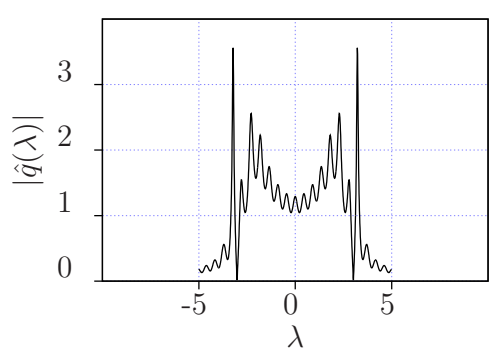

(a)

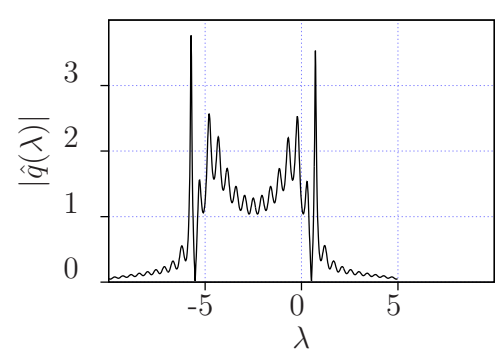

(b)

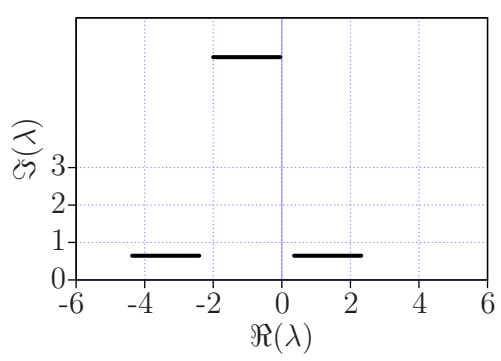

(c)

Fig. 13. (a) Amplitude of the continuous spectrum with no carrier. (b) Amplitude of the continuous spectrum with carrier frequency $\omega=5$. The phase graph is also shifted similarly with no other change $(\Delta \lambda=2.5)$. (c) Locus of the eigenvalues of a sinc function with amplitude $A=8$ as the carrier frequency $\omega$ in $\exp (-j \omega t)$ varies.

$\lambda_{1}=1.4234 j$ and a new pair of eigenvalues emerges, starting from $\lambda_{23}= \pm 3.2+0.05 j$. When the newly created eigenvalues are not pronounced enough, for instance in this example when transiting from $A=\pi$ to $A=1.27 \pi$, numerical algorithms have difficulties in determining whether these small emerging eigenvalues are part of the spectrum or not. Here it appears that for $\pi<A \leq 1.27 \pi$ there is just one dominant purely imaginary eigenvalue moving upward. At $A=1.27 \pi, \lambda_{23}$ emerge and move up in the complex plane as $A$ is increased. An important observation is that the sinc function appears to have not only purely imaginary eigenvalues, but also a pair of symmetric eigenvalues with nonzero real part emerging at high values of $A$; see Fig. 11 b). This means that, for example, a sinc function (viewed in the time domain) contains a stationary "central component" plus two small "side components" which travel to the left and right if the sinc function is subject to the NLS flow. The locus of the eigenvalues of the function $A \operatorname{sinc}(2 t)$ as a result of variations in $A$ is given in Fig. 11

It follows that the (truncated) sinc function is a simple example of a real symmetric signal whose eigenvalues are not necessarily purely imaginary, as conjectured for a long time [17]. However if $q(t)$ is real, non-negative, and "single-lobe", then there are exactly $N=\left\lfloor\frac{1}{2}+\frac{\|q\|_{L_{1}}}{\pi}-\epsilon\right\rfloor$ eigenvalues, all purely imaginary [17].

Remark 1. It appears that the (truncated) sinc function has a large number of eigenvalues distributed around the real axis. Since these eigenvalues have small imaginary parts and there are too many of them, sometimes it is hard to tell if they are spurious or part of the spectrum. This portion of the spectrum can be sensitive to the number of signal samples $N$ and the truncation window as well. One should thus make sure that $N$ is large enough and eigenvalues are computed and compared using both search and matrix-based methods. Generally speaking, only dominant eigenvalues with large enough imaginary parts can be trusted. The continuous spectrum also appears somewhat oscillatory. As a result, sinc functions do not appear to have desirable nonlinear spectrum.

Under phase modulation, in the form of introducing a constant phase term to the signal, the eigenvalues and the magnitude of the continuous spectrum remained unchanged. Vertical (negative) shift in the phase of the continuous spectrum as a result of phase modulation can be seen in Fig. 12

We may also examine the effect of time-dependent phase
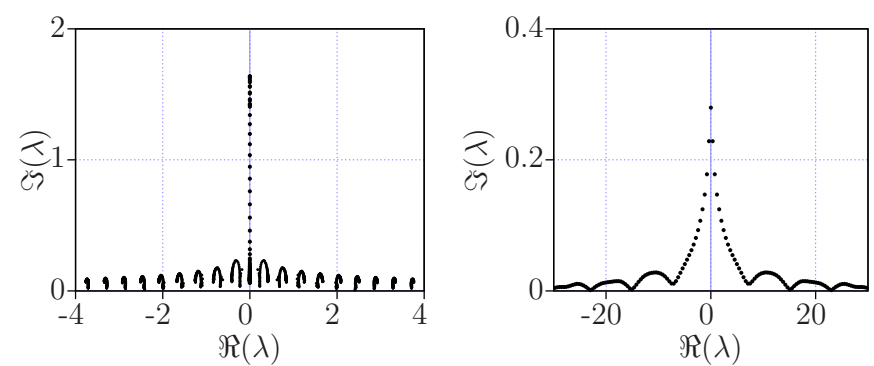

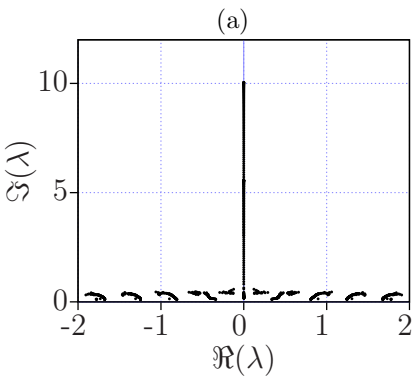

(c)

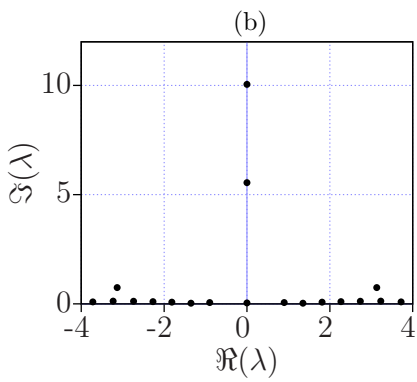

(d)
Fig. 14. Eigenvalues of $A e^{j \omega t^{2}} \operatorname{sinc}(2 t)$ : (a) locus of eigenvalues for $A=4$ and $0.5 \leq \omega \leq 50$, (b) eigenvalues for $A=4$ and $\omega=15$, (c) locus of eigenvalues for $A=12$ and $0 \leq \omega \leq 50$, (d) eigenvalues for $A=12$ and $\omega=0.50$

changes. The effect of linear chirp, of the form $\exp (j \omega t)$, is shown in picture Fig. 13 Linear chirp results in just a shift of the discrete and continuous spectrum to the left or the right, depending on the sign of the chirp.

It is interesting to observe the effect of a quadratic chirp. The locus of eigenvalues that result due to changes in the quadratic phase $q \exp \left(j \omega t^{2}\right)$ has been studied in [17] for Gaussian pulses. In our sinc function example, in the case that there is one discrete eigenvalue in the chirp-free case (such as when $A=4$ ), increasing $\omega$ will move the eigenvalue on the $j \omega$ axis upward, but then the eigenvalue moves down again and is absorbed in the real axis; see Figs. 14(a)-(b). Note that the eigenvalues off the $j \omega$ axis with small imaginary parts are considered to be spurious; their number increases as the number of sample points is increased.

A more interesting behavior is observed when $A=12$. Here, there are two eigenvalues on the $j \omega$ axis: $\lambda_{1} \approx 10.05 j$, $\lambda_{2}=5.55 j$, together with $\lambda_{3,4}= \pm 3.1+0.18 j$ (Fig 14(d)). As $\omega$ is increased from zero, $\lambda_{1}$ and $\lambda_{2}$ move down and a fifth 


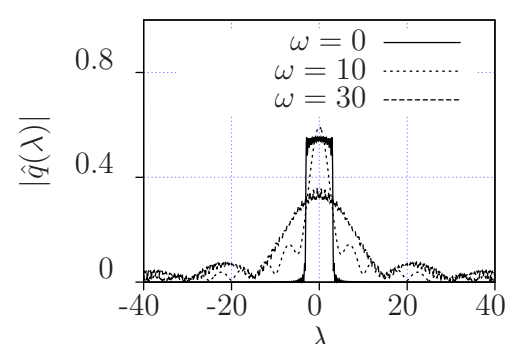

(a)

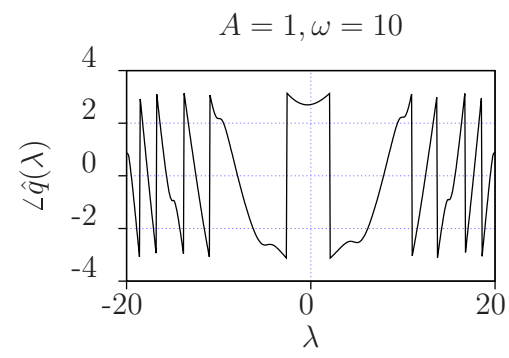

(b)

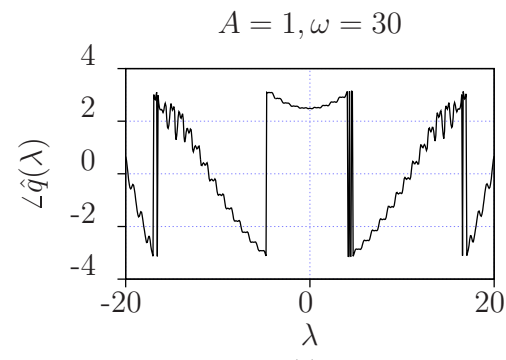

(c)

Fig. 15. (a) Nonlinear spectral broadening as a result of quadratic phase modulation $A e^{j \omega t^{2}} \operatorname{sinc}(2 t)$ with $A=1$ and $\omega=0,10$ and 30 . (b) Phase of the continuous spectrum when $A=1$ and $\omega=10$. (c) Phase of the continuous spectrum when $A=1$ and $\omega=30$.

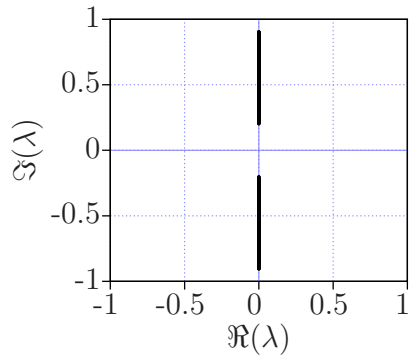

(a)

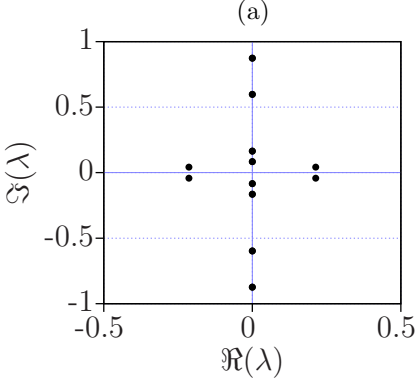

(c)

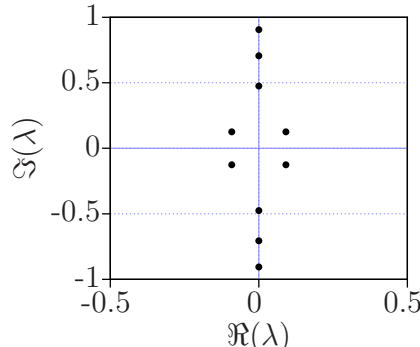

(b)

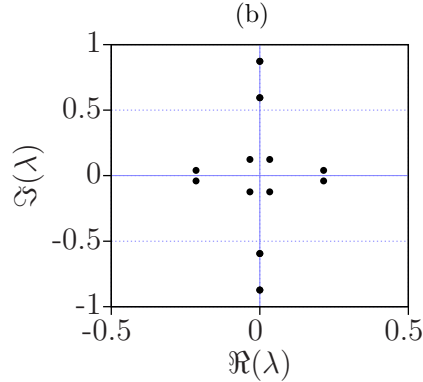

(d)
Fig. 16. Locus of eigenvalues of $\operatorname{sinc}(a t)$ as the bandwidth $a$ varies (a) from $a=0.1$ to $a=0.6$. (Eigenvalues with small $\Im \lambda$ are not shown here.) (b) Eigenvalues for $a=0.1$. (c) Eigenvalues before collision and (d) after collision.

eigenvalue $\lambda_{5}$ emerges from the real axis and moves upwards on the $j \omega$ axis. It appears that at about $\omega=41.41, \lambda_{2}$ and $\lambda_{5}$ "collide" and move out of the $j \omega$ axis to the left and right. If $\omega$ is further increased, $\lambda_{2}$ and $\lambda_{5}$ are absorbed into the real axis; see Figs. 14 (c)-(d).

The locus of eigenvalues as a function of the bandwidth can be studied similarly. Signal $q(t)=\operatorname{sinc}(a t)$ has 3 eigenvalues on the $j \omega$ axis for $a=0.1$, plus two small eigenvalues on two sides of the $j \omega$ axis (Fig 16 b)). As $a$ is increased, the smaller eigenvalue on the $j \omega$ axis comes down and a new eigenvalue is generated at the origin, moving upward. These two eigenvalues collide at $0.12 j(a=0.1330)$ and are diverted to the first and second quadrant, and eventually absorbed in the real axis at about $\Re \lambda= \pm 0.32(a=0.1990)$, Figs. 16(c)-(d)). As $a$ is decreased, more eigenvalues appear on the $j \omega$ axis and fewer on the real axis. Note that the eigenvalues are not necessarily on the $j \omega$ axis. For example, the signal $y=\operatorname{sinc}(0.1370 t)$ clearly has eigenvalues $\lambda_{1}=0.8684 j, \lambda_{2}=0.5797 j, \lambda_{3,4}=$ $\pm 0.1055+0.1210 i$.

Fig. 15 shows the nonlinear spectrum of a sinc function

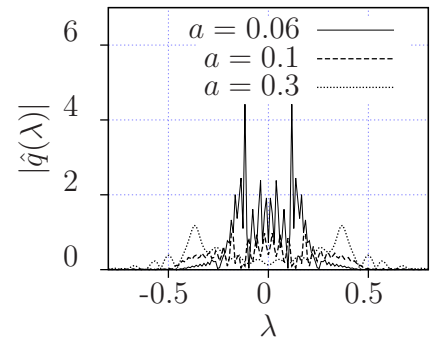

(a)

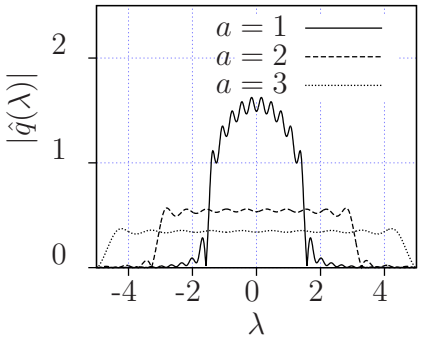

(b)
Fig. 17. Bandwidth expansion in $\operatorname{sinc}(a t)$ for (a) $a=0.06, a=0.1$, $a=0.3$ (b) $a=1, a=2, a=3$.

under a quadratic chirp modulation, given by $A e^{j \omega t^{2}} \operatorname{sinc}(2 t)$, is broadened as $\omega$ varies.

The effect of time dilation on the continuous spectrum can be seen in Fig 17. It can be observed that increasing bandwidth $a$, will increase the continuous range of real nonlinear frequencies, leading to bandwidth expansion.

2) Sinc Wavetrains: The nonlinear spectrum of a wavetrain can take on a complicated form, just like its ordinary Fourier transform counterpart. Eigenvalues of a two-symbol train, for instance, depend on the amplitude and phase of the two signals, and their separation distance.

We first analyze the case in which there are only two sinc functions located at the fixed Nyquist distance from each other, i.e., $q(t)=a_{1} \operatorname{sinc}(2 t-1)+a_{2} \operatorname{sinc}(2 t+1)$. For $a_{1}=a_{2}=2$ the spectrum consists of $\lambda_{1}=0.3676 j, \lambda_{2,3}= \pm 2.5+0.03 j$ and a number of spurious eigenvalues as shown in Fig. 18. a). As the phase of $a_{2}$ is increased from $\theta=0$ to $\theta=\pi, \lambda_{1}$ moves off the $j \omega$ axis to the left and a new eigenvalue emerges from the real axis in the first quadrant. Eigenvalues at $\theta=\pi$ are $\pm 1.3908+0.3287 i$. The resulting locus of eigenvalues is shown in Fig. 18(b). Figs. 18 (c)-(d) depict similar graphs when $a_{1}$ or $a_{2}$ change.

Next we study the locus of the discrete spectrum as a function of pulse separation for fixed amplitudes. If the amplitude of the sinc functions is increased sufficiently, eigenvalues appear off the real axis and form a locus as the distance between pulses varies. Fig. 19 (a) shows the locus of eigenvalues of $q(t)=4 \operatorname{sinc}(2 t+2 \tau)+4 \operatorname{sinc}(2 t-2 \tau)$ as $\tau$ changes between zero to 5 . At $\tau=0$, eigenvalues are $\lambda_{1}=6 j$, $\lambda_{2,3}= \pm 2.3618+0.6476 i$ and $\lambda_{4,5}= \pm 3.2429+0.0815 i$. As the distance between pulses is increased, $\lambda_{1}$ rapidly decreases, 


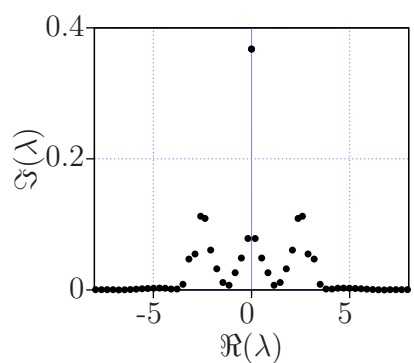

(a)

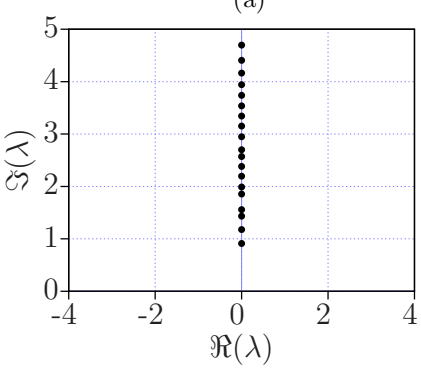

(c)

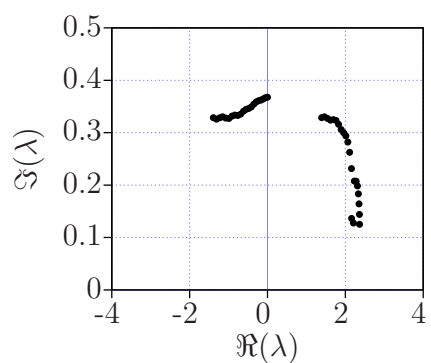

(b)

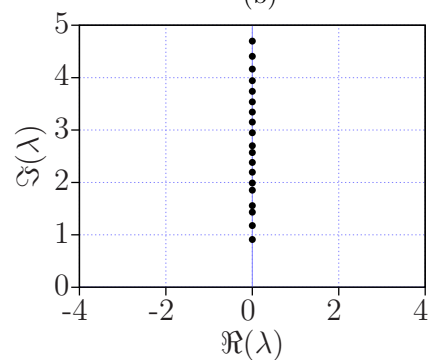

(d)

Fig. 18. Discrete spectrum of $q(t)=a_{1} \operatorname{sinc}(2 t-1)+a_{2} \operatorname{sinc}(2 t+1)$ for (a) $a_{1}=a_{2}=2$, (b) $a_{1}=2, a_{2}=2 e^{j \theta}$ for $-\pi<\theta \leq \pi$, (c) $a_{1}=2$, $0 \leq a_{2} \leq 6$, (d) $a_{1}=4 j, 0 \leq a_{2} \leq 6$.

and at about $\tau=0.2$, where $\lambda_{1}=4.27 j$, the eigenvalues with non-zero real parts are absorbed into the real axis at $\Re \lambda=-3$. As $\tau$ is further increased, $\lambda_{1}$ decreases further, until $\tau=0.4$ where $\lambda_{1}=2.12 j$ and two new eigenvalues emerge at locations $\Re \lambda= \pm 1.5$ going up and towards the $j \omega$ axis. There are also a large number of eigenvalues with small imaginary parts which arise from the real axis but return, before reaching the $j \omega$ axis, to be absorbed into the real axis, while new similar eigenvalues are generated again from the real axis. At $\tau=0.7$ eigenvalues are $\lambda=2 j, \pm 1+j$. At some point, the newly created eigenvalues are not absorbed into the real axis, but they reach the $j \omega$ axis and collide. For instance, at $\tau=1$ the two eigenvalues off the $j \omega$ axis rapidly travel towards the $j \omega$ axis and collide at $\tau=1.05$. One of these eigenvalues goes down to be absorbed into the origin, and the other one, interestingly, goes up to be united with the maximum eigenvalue on the $j \omega$ axis (i.e., to create one eigenvalue with multiplicity two). Increasing the distance further does not change the location of this eigenvalue, which from now is fixed at $\lambda=1.4 j$, but just changes the pattern of lower level eigenvalues. At this point $(\tau=5)$, the two sinc functions are separated in time. The collision does not occur when the amplitudes of the signals are smaller; see Fig. 19 (b) for the locus of the eigenvalues when the amplitude of the two sinc functions is 2 .

Remark 2. When eigenvalues seem to collide or unite together, the continuous spectrum develops delta-like spikes in its graph. This singularity (ill-conditioned eigenproblem) makes it difficult to determine eigenvalues accurately.

For wavetrains with a larger number of signals, the number of eigenvalues increases proportionally. We generate these wavetrains randomly and examine the region to which the spectrum is confined. Fig. 20 shows the locus of the discrete

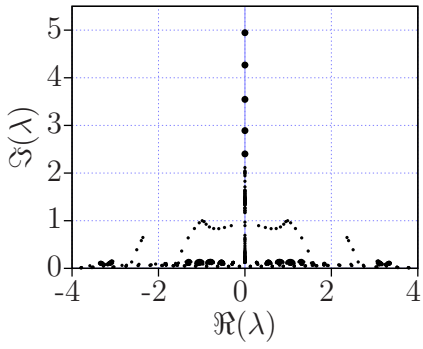

(a)

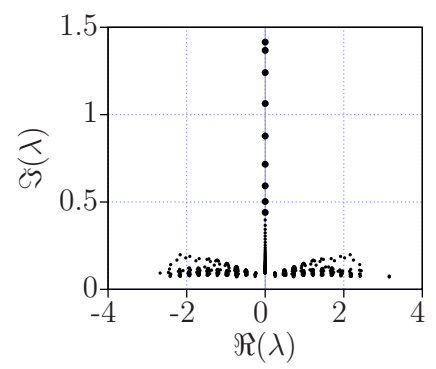

(b)
Fig. 19. (a) The locus of the discrete spectrum of $q(t)=4 \operatorname{sinc}(2 t+2 \tau)+$ $4 \operatorname{sinc}(2 t-2 \tau)$ as a function of $0 \leq \tau \leq 5$. (b) The locus of the discrete spectrum of $q(t)=2 \operatorname{sinc}(2 t+2 \bar{\tau})+\overline{2} \operatorname{sinc}(2 t-2 \tau)$ as a function of $0 \leq \tau \leq 5$.

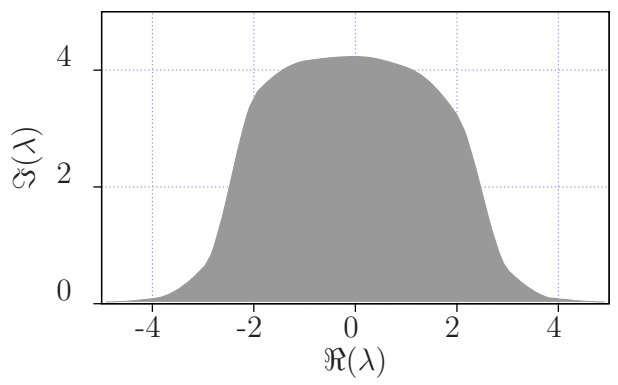

Fig. 20. Effect of the bandwidth constraint on the location of the eigenvalues of a sinc wavetrain containing 16 pulses with random amplitudes.

spectrum of all sinc wavetrains with 16 signals. All 16 signal degrees-of-freedom in the bandlimited signal are modulated here. The effect of the bandwidth constraint in the nonlinear spectral domain can seen in this picture.

\section{B. Preservation of the Spectrum}

It is crucial to ensure that the spectrum found by the numerical methods, such as those discussed in the previous sections, is in fact correct. While it proved difficult to do so consistently and efficiently, there are various tests to increase one's confidence in the truth of the output of the numerical methods. Taking the inverse nonlinear Fourier transform in the continuous-time domain and comparing the resulting function in time with the original signal is generally quite cumbersome and not always feasible. One quick test is to examine a time frequency identity, such as the trace formula for $n=1,2,3, \ldots$ as used in this paper. The first few conserved quantities in this identity can be written explicitly. One should allow higher tolerance values in the trace formula for large $n$, as the discrete terms in this identity involve $\lambda^{n}$ and thus are increasingly more sensitive to the eigenvalues. Another test is to subject the signal to the flow of an integrable equation, such as the NLS equation, and check that the discrete spectrum is preserved and the spectral amplitudes are scaled appropriately according to that equation. In this section, we let the signal propagate according to the NLS equation and compare the spectra at $z=0$ and $z=\mathcal{L}$ for various $\mathcal{L}$.

Fig. 21 shows examples of the spectra of a number of signals at $z=0$ and $z=\mathcal{L}$ evolving according to the NLS equation (1). The distances mentioned in the graphs in $\mathrm{km}$ correspond 

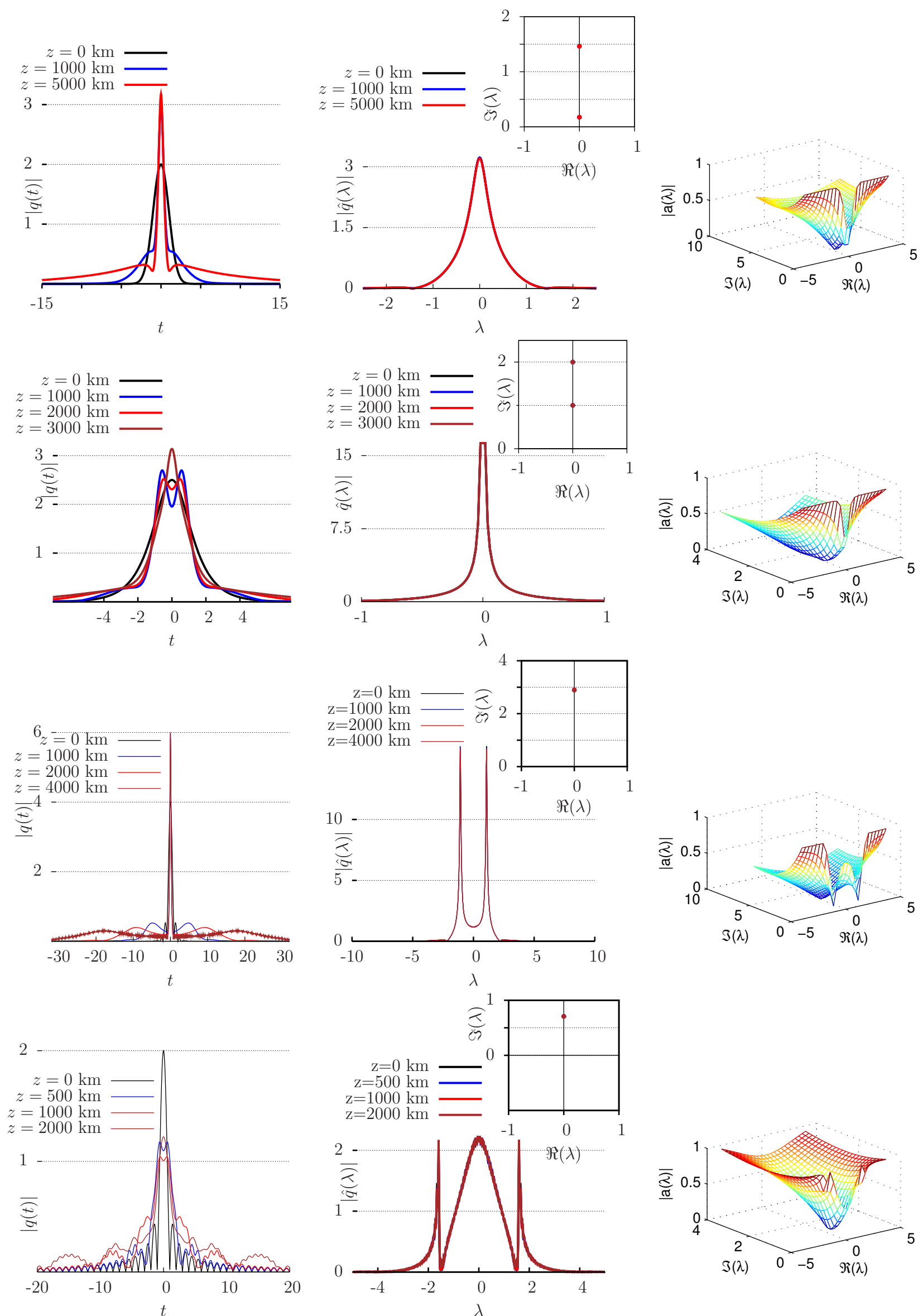

Fig. 21. Propagation of signals along an optical fiber in the time domain (left), in the nonlinear Fourier transform domain (middle), and showing the surface of $|a(\lambda)|$ (right). The signals are (a) Gaussian signal, (b) Satsuma-Yajima signal, (c) raised-cosine function, (d) sinc function. The zeros of $|a(\lambda)|$ correspond to eigenvalues in $\mathbb{C}^{+}$. 
TABLE I

Fiber PARAMETERS

\begin{tabular}{l|l|l}
$D$ & $17 \mathrm{ps} /(\mathrm{nm}-\mathrm{km})$ & $\begin{array}{l}\text { Dispersion parameter } \\
\text { nonlinearity parameter }\end{array}$ \\
$\gamma$ & $1.27 \mathrm{~W}^{-1} \mathrm{~km}^{-1}$ &
\end{tabular}

to a standard optical fiber with parameters in Table [] Note that in all these examples the discrete spectrum is completely preserved, and the continuous spectral amplitudes undergo a phase change properly. Compared to Gaussian and raisedcosine examples, whose nonlinear Fourier transform can be found easily, the discrete spectrum of sinc functions is much more challenging to find. This is because the non-dominant eigenvalues off the $j \omega$ axis have small imaginary parts for typical parameters and are not sufficiently distinguished. They also have large real parts, increasing the search region. Sinc functions are thus not the best examples to illustrate the application of the NFT in optical fibers. We studied these ideal signals primarily because of their fundamental utility in linear digital communications.

\section{CONCLUSIONS}

In this paper, we have suggested and compared a variety of numerical methods for the computation of the nonlinear Fourier transform of a signal defined on the entire real line. A straightforward finite-difference discretization, such as the forward discretization, does not often produce satisfactory results. Among the methods studied in this paper, the layerpeeling and spectral methods gave accurate results in estimating the continuous and discrete spectrum over a wide class of examples.

Given a waveform without having prior knowledge of the location of the discrete eigenvalues, we suggest the use of matrix-based methods to compute the discrete spectrum. If, on the other hand, the location of the eigenvalues is known approximately (as in data-communication problems, where the eigenvalues are chosen at the transmitter from a finite set) a search-based method is recommended.

Although the eigenvalues and the continuous spectral function can be calculated with great accuracy, the discrete spectral amplitudes are quite sensitive to the location of the eigenvalues, even in the absence of noise. The discrete spectral amplitudes control the time center of the signal, and are therefore sensitive to timing jitter. For data communication purposes it follows that, whereas the presence or absence of the eigenvalue itself may allow for robust information transmission, encoding information in the time center of the signal, i.e., in the discrete spectral amplitudes (in the RiemannHilbert approach), is unlikely to be viable.

Using these numerical methods, we studied the influence of various signal parameters on the nonlinear Fourier transform of a number of signals commonly used in data communications. We found, for example, that the spectrum of an isolated normalized sinc function with amplitude $A$ is purely continuous for the $A<\pi$. However, as the signal amplitude is increased, dominant eigenvalues appear on the $j \omega$ axis, together with pairs of symmetric eigenvalues having nonzero real parts.
In general, amplitude variations result in variations in the location of the eigenvalues and the shape of the continuous spectrum. Eigenvalues follow particular trajectories in the complex plane. Phase variations, on the other hand, influence only the phase of the spectrum, not the location of the eigenvalues. One important observation, which may be beneficial for the design of data communication systems, is that the nonlinear spectrum of bandlimited signals appears to be confined to a vertical strip in the complex plane with a width proportion to the signal bandwidth.

This paper has only scratched the surface of a potentially rich research area. The development of efficient and robust numerical techniques suitable for various engineering applications of the nonlinear Fourier transform will require significant additional effort. A problem of particular interest is the development of a "fast" nonlinear Fourier transform method that would be the analog of the FFT.

\section{REFERENCES}

[1] M. I. Yousefi and F. R. Kschischang, "Information transmission using the nonlinear Fourier transform, Part I: Mathematical tools," Arxiv e-prints, arXiv:1202.3653, Feb. 2012. [Online]. Available: http://arxiv.org/abs/1202.3653

[2] M. J. Ablowitz, D. J. Kaup, A. C. Newell, and H. Segur, "The inverse scattering transform - Fourier analysis for nonlinear problems," Stud. Appl. Math., vol. 53, no. 4, pp. 249-315, Dec. 1974.

[3] J. Stoer and R. Bulirsch, Introduction to Numerical Analysis, 3rd ed., ser. Texts Appl. Math. New York, NY, USA: Springer-Verlag, 2002, vol. 12 .

[4] D. W. McLaughlin and C. M. Schober, "Chaotic and homoclinic behavior for numerical discretizations of the nonlinear Schrödinger equation,' Physica D: Nonl. Phenom., vol. 57, no. 3-4, pp. 447-465, Aug. 1992.

[5] C. P. Olivier, "A numerical study of the spectrum of the nonlinear Schrödinger equation," Master's thesis, Dept. Math., Stellenbosch U., Stellenbosch, South Africa, Dec. 2008.

[6] T. Tao and C. Thiele, "Nonlinear Fourier analysis," Arxiv e-prints, arXiv:1201.5129, Jan. 2012. [Online]. Available: http://arxiv.org/abs/1201.5129

[7] A. R. Osborne, Nonlinear Ocean Waves and the Inverse Scattering Transform, 1st ed., ser. Int. Geophys. New York, NY, USA: Academic Press, 2010, vol. 97.

[8] J. Skaar and O. H. Waagaard, "Design and characterization of finitelength fiber gratings," IEEE J. Quantum Electron., vol. 39, no. 10, pp. 1238-1245, Oct. 2003.

[9] M. J. Ablowitz and J. F. Ladik, "Nonlinear differential-difference equations and Fourier analysis," J. Math. Phys., vol. 17, no. 6, pp. 10111018, Jun. 1976.

[10] M. J. Ablowitz and H. Segur, Solitons and the Inverse Scattering Transform, ser. SIAM Stud. Appl. Numer. Math. Philadelphia, PA, USA: SIAM, 1981, vol. 4.

[11] T. R. Taha and M. J. Ablowitz, "Analytical and numerical aspects of certain nonlinear evolution equations. II. Numerical, nonlinear Schrödinger equation," J. Comput. Phys., vol. 55, no. 2, pp. 203-230, Aug. 1984.

[12] J. H. Wilkinson, The Algebraic Eigenvalue Problem. Oxford, U.K.: Oxford University Press, 1965.

[13] J. Wilkinson and C. Reinsch, Handbook for Automatic Computation, Vol. 2: Linear Algebra, ser. Grundlehren der mathematischen Wissenschaften. New York, NY, USA: Springer-Verlag, 1971, vol. 186.

[14] A. R. Bishop, D. W. McLaughlin, M. G. Forest, and E. A. Overman II, "Quasi-periodic route to chaos in a near-integrable PDE: Homoclinic crossings," Phys. Lett. A, vol. 127, no. 6-7, pp. 335-340, Mar. 1988.

[15] M. J. Ablowitz and B. M. Herbst, "On homoclinic structure and numerically induced chaos for the nonlinear Schrödinger equation," SIAM J. Appl. Math., vol. 50, no. 2, pp. 339-351, Apr. 1990.

[16] J. Satsuma and N. Yajima, "B. Initial value problems of one-dimensional self-modulation of nonlinear waves in dispersive media," Prog. Theoret. Phys. Suppl., no. 55, pp. 284-306, Jan. 1974.

[17] M. Klaus and J. Shaw, "On the eigenvalues of Zakharov-Shabat systems," SIAM J. Math. Anal., vol. 34, no. 4, pp. 759-773, Feb. 2003. 